\title{
THE BLAST SURVEY OF THE VELA MOLECULAR CLOUD: PHYSICAL PROPERTIES OF THE DENSE CORES IN VELA-D
}

\author{
Luca Olmi ${ }^{1,2}$, Peter A. R. Ade ${ }^{3}$, Daniel Anglés-Alcázar ${ }^{1,4}$, James J. Bock $^{5,6}$, Edward L. Chapin ${ }^{7}$, Massimo De Luca $^{8}$, \\ Mark J. Devlin ${ }^{9}$, Simon Dicker ${ }^{9}$, Davide Elia ${ }^{10}$, Giovanni G. Fazio ${ }^{11}$, Teresa Giannini ${ }^{12}$, MatThew Griffin $^{3}$, \\ Joshua O. Gundersen ${ }^{13}$, Mark Halpern ${ }^{7}$, Peter C. Hargrave ${ }^{3}$, David H. Hughes ${ }^{14}$, JefF Klein ${ }^{9}$, Dario Lorenzetti ${ }^{12}$, \\ Massimo Marengo $^{11}$, Gaelen Marsden ${ }^{7}$, Peter G. Martin ${ }^{15}, 16$, Fabrizio Massi ${ }^{2}$, Philip Mauskopf ${ }^{3}$, \\ Calvin B. Netterfield ${ }^{16,17}$, Guillaume Patanchon ${ }^{18}$, Marie Rex $^{9}$, Alberto Salama $^{19}$, Douglas Scott $^{7}$, \\ Christopher Semisch ${ }^{9}$, Howard A. Smith ${ }^{11}$, Francesco Strafella ${ }^{20}$, Nicholas Thomas ${ }^{13}$, Matthew D. P. Truch ${ }^{9}$, \\ Carole Tucker $^{3}$, Gregory S. Tucker ${ }^{21}$, Marco P. Viero ${ }^{16}$, and Donald V. Wiebe ${ }^{17}$ \\ ${ }^{1}$ University of Puerto Rico, Rio Piedras Campus, Physics Department, Box 23343, UPR station, San Juan, Puerto Rico, USA; olmi.luca@gmail.com, \\ olmi@arcetri.astro.it \\ ${ }^{2}$ INAF, Osservatorio Astrofisico di Arcetri, Largo E. Fermi 5, I-50125, Firenze, Italy. \\ ${ }^{3}$ Department of Physics \& Astronomy, Cardiff University, 5 The Parade, Cardiff, CF24 3AA, UK \\ ${ }^{4}$ Department of Physics, University of Arizona, 1118 E. 4th Street, Tucson, AZ 85721, USA \\ 5 Jet Propulsion Laboratory, Pasadena, CA 91109-8099, USA \\ ${ }^{6}$ Observational Cosmology, MS 59-33, California Institute of Technology, Pasadena, CA 91125, USA \\ ${ }^{7}$ Department of Physics \& Astronomy, University of British Columbia, 6224 Agricultural Road, Vancouver, BC V6T 1Z1, Canada \\ ${ }^{8}$ LERMA-LRA, CNRS UMR8112, Observatoire de Paris and Ecole Normale Supérieure, 24 Rue Lhomond, 75231 Paris cedex 05, France \\ ${ }^{9}$ Department of Physics and Astronomy, University of Pennsylvania, 209 South 33rd Street, Philadelphia, PA 19104, USA \\ ${ }^{10}$ Universidade de Lisboa - Faculdade de Ciencias, Centro de Astronomia e Astrofísica da Universidade de Lisboa, Observatório Astronómico de Lisboa, \\ Tapada da Ajuda, 1349-018 Lisboa, Portugal \\ ${ }^{11}$ Harvard-Smithsonian Center for Astrophysics, Cambridge, MA 02138, USA \\ ${ }^{12}$ INAF, Osservatorio Astronomico di Roma, Via Frascati 33, I-00040 Monteporzio Catone, Roma, Italy \\ ${ }^{13}$ Department of Physics, University of Miami, 1320 Campo Sano Drive, Carol Gables, FL 33146, USA \\ ${ }^{14}$ Instituto Nacional de Astrofísica Óptica y Electrónica (INAOE), Aptdo. Postal 51 y 72000 Puebla, Mexico \\ ${ }^{15}$ Canadian Institute for Theoretical Astrophysics, University of Toronto, 60 St. George Street, Toronto, ON M5S 3H8, Canada \\ ${ }^{16}$ Department of Astronomy \& Astrophysics, University of Toronto, 50 St. George Street, Toronto, ON M5S 3H4, Canada \\ ${ }^{17}$ Department of Physics, University of Toronto, 60 St. George Street, Toronto, ON M5S 1A7, Canada \\ ${ }^{18}$ Laboratoire APC, 10, rue Alice Domon et Léonie Duquet 75205 Paris, France \\ ${ }^{19}$ European Space Astronomy Centre, Villanueva de la Canada, Apartado 78, 28691 Madrid, Spain \\ ${ }^{20}$ Dipartimento di Fisica, Univ. del Salento, CP 193, I-73100 Lecce, Italy \\ ${ }^{21}$ Department of Physics, Brown University, 182 Hope Street, Providence, RI 02912, USA \\ Received 2009 September 2; accepted 2009 November 3; published 2009 December 7
}

\begin{abstract}
The Balloon-borne Large-Aperture Submillimeter Telescope (BLAST) carried out a 250, 350, and $500 \mu \mathrm{m}$ survey of the galactic plane encompassing the Vela Molecular Ridge, with the primary goal of identifying the coldest dense cores possibly associated with the earliest stages of star formation. Here, we present the results from observations of the Vela-D region, covering about $4 \mathrm{deg}^{2}$, in which we find 141 BLAST cores. We exploit existing data taken with the Spitzer MIPS, IRAC, and SEST-SIMBA instruments to constrain their (single-temperature) spectral energy distributions, assuming a dust emissivity index $\beta=2.0$. This combination of data allows us to determine the temperature, luminosity, and mass of each BLAST core, and also enables us to separate starless from protostellar sources. We also analyze the effects that the uncertainties on the derived physical parameters of the individual sources have on the overall physical properties of starless and protostellar cores, and we find that there appear to be a smooth transition from the pre- to the protostellar phase. In particular, for protostellar cores we find a correlation between the MIPS24 flux, associated with the central protostar, and the temperature of the dust envelope. We also find that the core mass function of the Vela-D cores has a slope consistent with other similar (sub)millimeter surveys.
\end{abstract}

Key words: balloons - ISM: clouds - stars: formation - submillimeter

Online-only material: color figures, machine-readable tables

\section{INTRODUCTION}

Stars form in dense, dusty cores of molecular clouds, but little is known about their origin, their evolution, and their detailed physical properties. In particular, the relationship between the core mass function (CMF) and the stellar initial mass function (IMF) is poorly understood (McKee \& Ostriker 2007). One of the reasons for this lack of understanding, from the observational point of view, is the difficulty in selecting a statistically significant sample of truly pre-stellar cores from an otherwise unremarkable collection of high column density features.
Pre-stellar cores represent a very early stage of the star formation (SF) process, before collapse results in the formation of a central protostar. The physical properties of these cores can reveal important clues about their nature; mass, spatial distributions, and lifetime are important diagnostics of the main physical processes leading to the formation of the cores from the parent molecular cloud. In addition, a comparison of the CMF to the IMF may help to understand what processes are responsible for further fragmentation of the cores, and thus the determination of stellar masses. Therefore, large samples of bona-fide pre-stellar cores are important for comparison of observations with various SF models and scenarios. 
Two major technological advances have revolutionized the study of the mass distribution in molecular clouds and the characterization of the earliest phases of SF: the Infrared Astronomical Satellite (IRAS), and the advent of submillimeter bolometer arrays such as MAMBO, SIMBA, and SCUBA. (Sub)millimeter surveys have been vitally important for probing the nature of SF, and in particular have represented the only way of deriving statistically significant samples of pre- and protostellar objects. However, these instruments have probed the Rayleigh-Jeans tail of the spectral energy distribution (SED) of these cold objects, far from its peak. Therefore, these surveys have been limited by their relative inability to measure the temperature (e.g., Motte et al. 1998), producing large uncertainties in the derived luminosities and masses. Recent surveys with the MIPS instrument of the Spitzer Space Telescope are able to constrain the temperatures of warmer objects (Carey et al. 2005), but the youngest and coldest objects are potentially not detected, even in the long-wavelength Spitzer bands. Other ground-based surveys conducted at wavelengths $\lesssim 450 \mu \mathrm{m}$ have been affected by low sensitivity due to atmospheric conditions at short submillimeter wavelengths (e.g., Kirk et al. 2005; Wu et al. 2007).

The Balloon-borne Large-Aperture Submillimeter Telescope, BLAST (Pascale et al. 2008), carried out two long duration balloon science flights. During the first (BLAST05), BLAST mapped several galactic star-forming regions (Chapin et al. 2008; Truch et al. 2008). During the second science flight in 2006, BLAST06 mapped about $50 \mathrm{deg}^{2}$ in the Vela Molecular Ridge (VMR; Netterfield et al. 2009). Until the more extensive results from Herschel become available, BLAST is unique in its ability to detect and characterize cold dust emission from both starless and protostellar sources, constraining the temperatures of objects with $T \lesssim 25 \mathrm{~K}$ using its three-band photometry (250, 350 , and $500 \mu \mathrm{m}$ ) near the peak of the cold core SED.

The VMR is a giant molecular cloud complex within the galactic plane, in the area $260^{\circ} \lesssim l \lesssim 272^{\circ}$ and $-2^{\circ} \lesssim b \lesssim 3^{\circ}$, hence located outside the solar circle. It was first studied in detail by Murphy \& May (1991), using millimeter low-resolution observations in the $\mathrm{CO}(1-0)$ transition. They subdivided the VMR into four main regions, named A, B, C, and D. Liseau et al. (1992) further discussed the issue of distance, finding that clouds A, C, and D are at $700 \pm 200 \mathrm{pc}$, whereas B seems to be at $\sim 2000 \mathrm{pc}$. In this work, we have selected the Vela-D region because of the large amount of ancillary data available, both in the continuum, particularly in the mid- (MIR) and far-infrared (FIR), and also in terms of spectral line observations.

Liseau et al. (1992) and Lorenzetti et al. (1993) identified a number of VMR sites that host low- and intermediate-mass (proto)stars whose infrared SEDs are consistent with those of Class I sources. Later, Massi et al. (1999, 2000, 2003) analyzed NIR images of a sample of those sites, finding that the IRAS sources with $L_{\text {bol }}>10^{3} L_{\odot}$ are associated with small embedded young stellar clusters. Their IMF appears consistent with that of field stars and their age is of the order of $10^{6} \mathrm{yr}$ (Massi et al. 2006). Remarkably, cloud D exhibits a lack of massive (O-type) stars with respect to what is expected from a standard IMF (Massi et al. 2006).

New millimeter observations, both spectral line (Elia et al. 2007) and continuum (Massi et al. 2007), have shown that all the IRAS sources associated with embedded young clusters are also associated with one or more dense molecular cores, confirming the inferred young age of the clusters. On-going SF activity in Vela-D is also confirmed by the presence of collimated jets originating from objects embedded in some of the clusters (Lorenzetti et al. 2002; De Luca et al. 2007). A first census of young stellar objects (YSOs) throughout the area of cloud D mapped by Massi et al. (2007) and Elia et al. (2007), was carried out by Giannini et al. (2007) using Spitzer MIPS observations at 24 and $70 \mu \mathrm{m}$ (hereafter indicated as MIPS24 and MIPS70, respectively). Most of the millimeter cores identified by Massi et al. (2007) were found to be associated with red, cold objects. Cloud D appears therefore as an active star-forming region with both distributed and clustered SF in progress, hosting a large sample of young objects in different evolutionary stages.

In this paper, we use both BLAST and archival data to determine the SEDs, and thus physical parameters, of each source detected by BLAST. In Section 2, we describe the BLAST observations and also the archival data that have been used in this work. In Section 3, we describe the identification of millimeter, MIR and FIR counterparts of the BLAST cores, and also describe the multi-band photometry. This photometry is then used to construct the SED of each source in Section 4, which allow us to derive the physical parameters for starless and protostellar cores, discussed in Section 5. We finally draw our conclusions in Section 6.

\section{OBSERVATIONS}

\subsection{BLAST Observations}

BLAST06 is a $1.8 \mathrm{~m}$ Cassegrain telescope, whose underilluminated primary mirror has produced in-flight beams with FWHM of $36^{\prime \prime}, 42^{\prime \prime}$, and $60^{\prime \prime}$ at 250,350 , and $500 \mu \mathrm{m}$, respectively. The camera consists of three silicon-nitride "spider web" bolometer arrays (Turner et al. 2001) almost identical to those for SPIRE on Herschel (Griffin et al. 2008), with 149, 88, and 43 detectors at 250,350 , and $500 \mu \mathrm{m}$, respectively, organized in a hexagonal close-packed pattern. The raw BLAST data are reduced using a common pipeline. More details can be found in the BLAST05 papers, Pascale et al. (2008), Patanchon et al. (2008), and Truch et al. (2008). The absolute gain of the instrument (including antenna efficiency), determined from regular observations of the evolved star VY CMa, is measured with highly correlated absolute uncertainties of $10 \%, 12 \%$, and $13 \%$ at 250,350 , and $500 \mu \mathrm{m}$, respectively (Truch et al. 2009). Finally, maximum likelihood maps and noise estimates are made (Patanchon et al. 2008, Wiebe 2008).

The deepest map of the Galactic Plane obtained by BLAST06 spans $\sim 10$ deg in Galactic longitude and $\sim 5$ deg in Galactic latitude in the constellation Vela (including the VMR) which we will call the "Vela deep" map hereafter. The Vela deep map is part of a much larger $\left(\sim 200 \mathrm{deg}^{2}\right)$ but shallower map, called the "Vela wide" map, ${ }^{22}$ which is still unpublished. As noted in the Introduction, we will concentrate here on the Vela-D region, which for practical reasons we define as the area of the Vela deep map contained within $262.80<l<264.60$ and $-1.15<b<1$. 10 . In this area, we find a total of 141 sources from the BLAST catalog of Vela deep, shown in Figure 1 and described later in Section 3 and Section 5. The high signal-tonoise ratio $(\mathrm{S} / \mathrm{N})$ of the BLAST maps allows us to infer intrinsic source sizes even below the beam FWHM scale by deconvolving the BLAST beam from the measured source FWHM (using a Gaussian source surface brightness profile: see Section 3.1 and Netterfield et al. 2009). We find that the sizes from the fit are broader than the intrinsic beam size, with a typical diameter of

\footnotetext{
22 http://www.blastexperiment.info/
} 


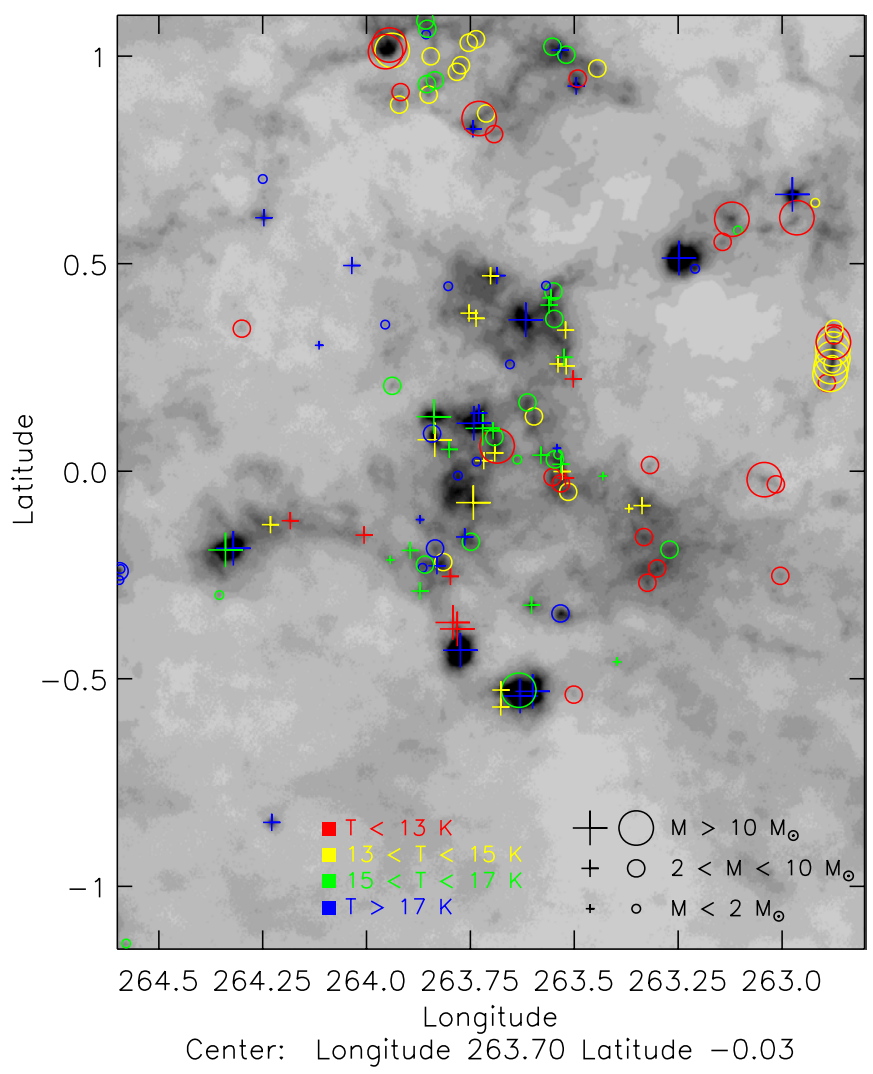

Figure 1. Gray-scale image shows the BLAST $250 \mu \mathrm{m}$ map of Vela-D, with galactic coordinates in degrees. Superimposed are the locations of both starless (open circles) and protostellar (crosses) cores (see Section 3 and Section 5). The size of both crosses and circles indicates the mass range of each core and color-coding indicates the core temperature (see legend). See Section 5.3 for a discussion of the cores' physical parameters.

(A color version of this figure is available in the online journal.)

62" (measured at $250 \mu \mathrm{m}$ ), which corresponds to an intrinsic deconvolved source size of $\simeq 0.15 \mathrm{pc}$ at the distance of the VMR.

\subsection{Archival Data}

\subsubsection{SEST Data}

Figure 2 shows the $1 \times 1 \mathrm{deg}^{2}$ area of Cloud D mapped with SIMBA at the SEST (ESO, La Silla, Chile) in the $1.2 \mathrm{~mm}$ continuum (Massi et al. 2007). At this wavelength, the SIMBA beam is $\simeq 24^{\prime \prime}$, i.e., $\sim 0.08 \mathrm{pc}$ at 700 pc. The rms in the map is in the range $14-40 \mathrm{mJy}$ beam $^{-1}$, but it is $\sim 20 \mathrm{mJy}^{\text {beam }}{ }^{-1}$ over most of the map (Massi et al. 2007). By using the algorithm CLUMPFIND (Williams et al. 1994), the emission was subdivided by Massi et al. (2007) into 29 dense molecular cores, with deconvolved sizes in the range $0.03-0.25 \mathrm{pc}$ and masses in the range $0.4-88 M_{\odot}$. Massi et al. (2007) discarded a total of 26 additional cores because they were smaller than the SEST beam. However, De Luca et al. (2007) showed that most of these objects are probably real millimeter sources associated with SF and not just instrumental artifacts. The two sets of objects and the assumptions made to derive their physical properties are discussed in Massi et al. (2007). Their Table 1 lists the robust sample of 29 sources (named "MMS" by the authors), while their Table 2 lists the sample of possible cores (named "umms"). Our own analysis of the SIMBA data and association with BLAST sources are discussed in Section 3.2.

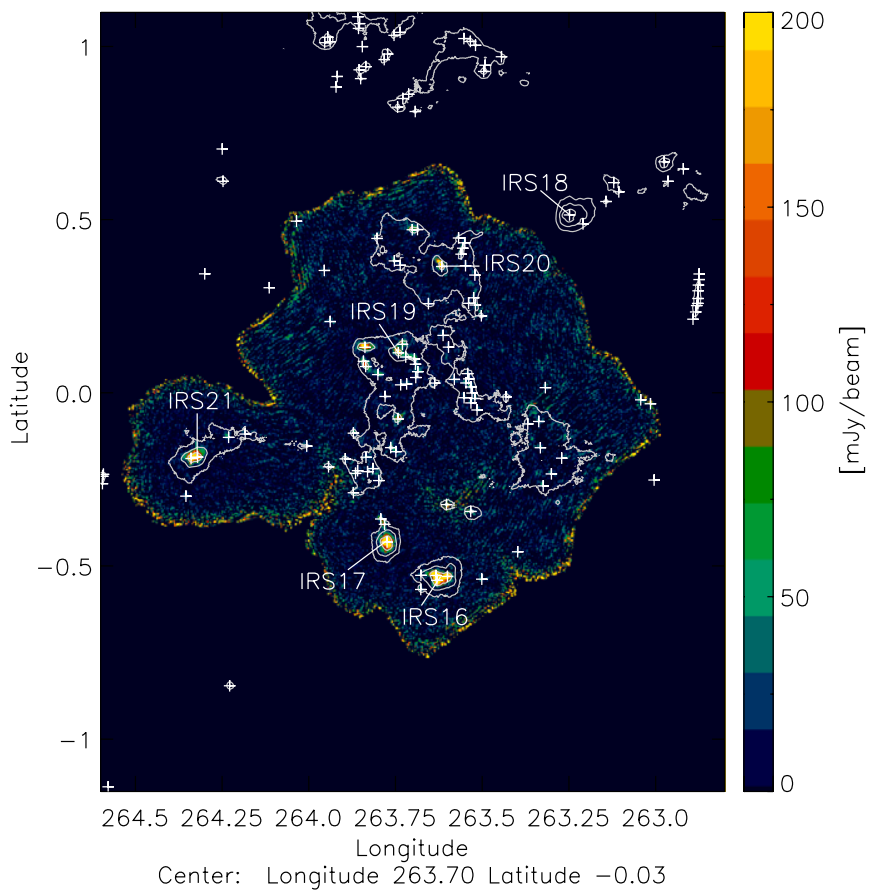

Figure 2. SIMBA $1.2 \mathrm{~mm}$ emission from Massi et al. (2007). Overplotted are the BLAST $250 \mu \mathrm{m}$ contours, and the positions of the compact BLAST sources ("+" signs). Also shown are the positions of the main bright infrared sources of Liseau et al. (1992). Galactic coordinates are in degrees.

(A color version of this figure is available in the online journal.)

\subsubsection{IRAC, MIPS, and Akari}

Concerning the Spitzer MIPS data (Rieke et al. 2004) in this paper, we make use of maps (covering $\sim 1.5 \mathrm{deg}^{2}$, see Figure 3) at 24 and $70 \mu \mathrm{m}$ of Vela-D which have already been published (Giannini et al. 2007), and the reader is referred to that paper where the image mosaicking, artifact removal and details relative to source finding and flux extraction (see also Section 3.3.2) are discussed.

To characterize the BLAST sources at the shortest possible wavelengths, we also use Spitzer IRAC (Fazio et al. 2004) observations, in particular those in the $8 \mu \mathrm{m}$ band, covering part of Vela-D (see Figures 3 and 4). The sensitivity achieved at $8 \mu \mathrm{m}$ was about $50 \mu \mathrm{Jy}$. Moreover, because Spitzer is diffraction limited longward of $\sim 10 \mu \mathrm{m}$, the spatial resolution in the MIPS bands is $\simeq 6^{\prime \prime}$ at $24 \mu \mathrm{m}$, and $\simeq 18^{\prime \prime}$ at $70 \mu \mathrm{m}$. We note that the present paper is based on a sub-sample of a much larger catalog incorporating both IRAC and MIPS data (Strafella et al. 2009).

The Akari/FIS survey at 60, 90, 140, and $160 \mu \mathrm{m}$ (Yamamura et al. 2008) is useful because of its much higher sensitivity compared to IRAS, and also because of the two longer wavelength bands, making it more sensitive to cold dust emission. An early Akari catalog has been used to find FIR counterparts to the BLAST sources, but without the actual maps we have not been able to directly compare the brightness distribution with that observed at both longer and shorter wavelengths.

\subsection{Morphology}

Figure 3 shows a false color image of the Vela-D map, and the $1.2 \mathrm{~mm}$ SIMBA map of the Vela-D region (Massi et al. 2007) is shown in Figure 2. The brightest regions in Figure 2 are clearly associated with known SF regions, including IRS16, IRS17, IRS18, IRS19, IRS20, and IRS21 (following the designation adopted by Liseau et al. 1992). IRS16 is located at the center of 


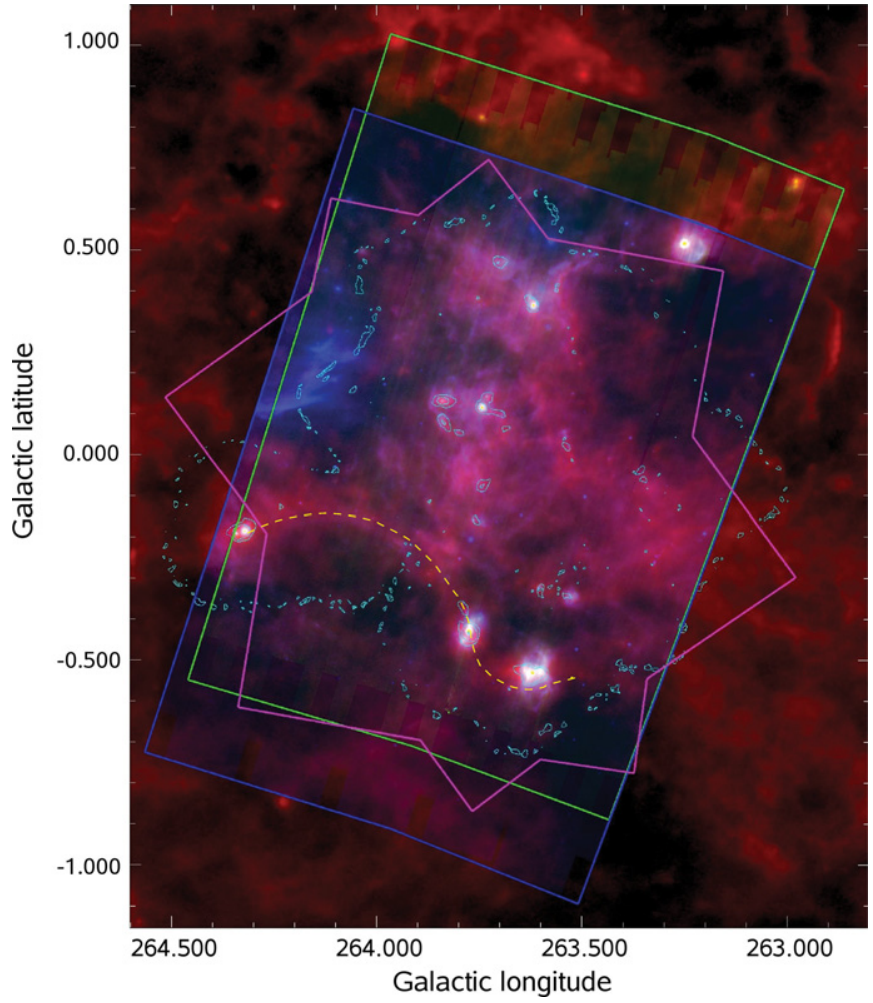

Figure 3. False color image of the Vela-D map using MIPS24 for blue, MIPS70 for green, and BLAST $250 \mu \mathrm{m}$ for red. Color in this image is an indicator of temperature, with blue regions usually being warmer and red regions being cooler. The background image is the BLAST $250 \mu \mathrm{m}$ map, the blue-shaded region represents the MIPS24 map, the region outlined by the green contour is the map covered by MIPS70, the irregular purple line outlines show the region covered by IRAC and the contours represent the SIMBA map. The " $S$ " shaped dashed, yellow contour shows the approximate location of the filament containing sources IRS16, IRS17 and IRS21 (see Section 2.3 and Figure 2).

(A color version of this figure is available in the online journal.)

the H II region 263.619-0.533 (Caswell \& Haynes 1987), and the most populated areas of the map with BLAST cores are clearly the regions around sources IRS19 and IRS20, which show a complex structure composed of filaments, diffuse emission and clusters of dense cores.

The nature of the IRS sources has been analyzed in detail by Massi et al. (2003), who concluded that most of these objects are probably precursors of intermediate mass stars $\left(M \sim 2-10 M_{\odot}\right)$ which may still be in an accretion stage (i.e., Class I sources), thus explaining their red colors. By comparing Figures 1 to 3, one can also see that IRS16, IRS17, and IRS21 are all part of a long, " $S$ "-shaped filament containing several BLAST and SIMBA cores, including the most massive SIMBA core, MMS4. The presence and nature of this, as well as other arc-like features in the Vela-D region, has been analyzed by Elia et al. (2007).

Figure 4 shows an overlay of the IRAC $8 \mu \mathrm{m}$ (hereafter IRAC8) image with the BLAST $250 \mu \mathrm{m}$ contours. Although both trace neutral material, the $8 \mu \mathrm{m}$ emission is known to be heavily influenced by polycyclic aromatic hydrocarbon (PAH) emission, and requires UV illumination, instead of being a simple tracer of column density. The IRAC8 map can also be used to check for extinction effects. In Figure 4, the BLAST contours follow the general distribution of the $8 \mu \mathrm{m}$ emission. However, there are a number of BLAST compact sources, along with the neutral material associated with them, that correspond in position with local minima or darker regions in the diffuse $8 \mu \mathrm{m}$ emission. This effect has also been observed in Vulpecula

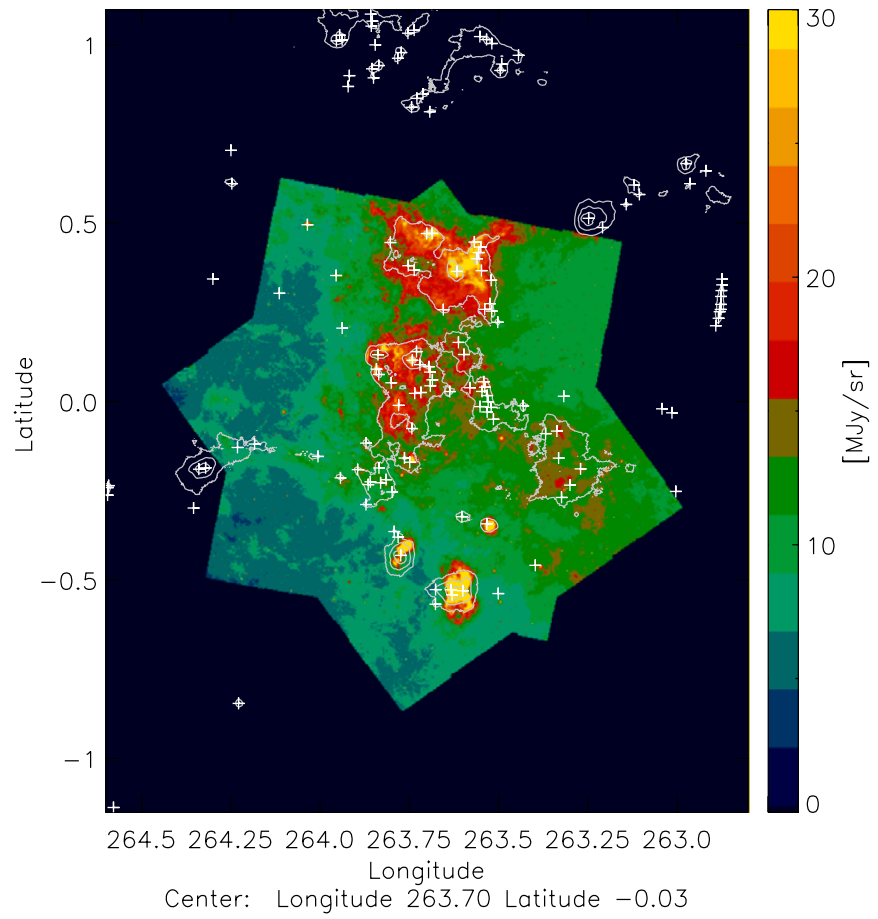

Figure 4. IRAC emission at $8 \mu \mathrm{m}$ overplotted with the BLAST $250 \mu \mathrm{m}$ contours and the positions of compact BLAST sources ("+" signs). We note that some of the BLAST sources occur at minima, or darker regions, in the diffuse $8 \mu \mathrm{m}$ emission, possibly indicating that the submillimeter objects are (associated with) infrared dark clouds in the foreground (see also Section 5.2). Galactic coordinates are in degrees.

(A color version of this figure is available in the online journal.)

by Chapin et al. (2008). However, analysing whether this is the result of chance coincidence or rather it is indeed the BLAST cores that are producing the so-called infrared dark clouds (IRDCs; e.g., Egan et al. 1998; Simon et al. 2006) was beyond the scope of this work.

Finally, we note that the BLAST cores are unlikely to be extragalactic bright sources. Using the number counts in Patanchon et al. (2009) the expected number of extragalactic sources at $250 \mu \mathrm{m}$ with flux density greater than $0.3 \mathrm{Jy}$ is $\simeq 1 \mathrm{deg}^{-2}$, while at the level of a few Jy (the observed minimum source flux density at $250 \mu \mathrm{m}$ ), this number is $\ll 1 \mathrm{deg}^{-2}$.

\section{SOURCE PHOTOMETRY AND ASSOCIATIONS WITH COMPACT SOURCES}

\subsection{Source and Flux Extraction in the BLAST Maps}

The source extraction and brightness estimation techniques applied to the BLAST maps here are similar to the method used during analysis of the BLAST05 data (Chapin et al. 2008). Candidate sources are identified by finding peaks after a Mexican Hat Wavelet type convolution (see, e.g., Barnard et al. 2004). The candidate lists from 250 and $350 \mu \mathrm{m}$ are then merged and fluxes at all three bands extracted by fitting a compact Gaussian profile to the source. Sources are not identified at $500 \mu \mathrm{m}$ due to the greater source-source and sourcebackground confusion resulting from the lower resolution. However, a Gaussian is refitted also in the $500 \mu \mathrm{m}$ map using the size and location parameters determined at the shorter wavelengths (the size of the Gaussian is convolved to account for the differing beam sizes). Using this technique, more than one thousand compact cores were identified in the Vela deep map (Netterfield et al. 2009). 
Table 1

BLAST Sources

\begin{tabular}{|c|c|c|c|c|c|c|c|c|c|c|}
\hline $\begin{array}{c}\text { Source } \\
\text { No. }\end{array}$ & Source Name & $\begin{array}{c}l \\
(\operatorname{deg})\end{array}$ & $\begin{array}{c}b \\
(\mathrm{deg})\end{array}$ & $\begin{array}{l}S_{250} \\
(\mathrm{Jy}) \\
\end{array}$ & $\begin{array}{l}S_{350} \\
(\mathrm{Jy}) \\
\end{array}$ & $\begin{array}{l}S_{500} \\
(\mathrm{Jy})\end{array}$ & $\begin{array}{c}T \\
(\mathrm{~K})\end{array}$ & $\begin{array}{c}M \\
\left(M_{\odot}\right)\end{array}$ & $\begin{array}{c}L \\
\left(L_{\odot}\right)\end{array}$ & $\begin{array}{c}\text { Deconvolved } \\
\text { Diameter } \\
(\mathrm{pc})\end{array}$ \\
\hline 0 & BLAST J084441-431144 & 263.0044 & -0.2518 & $7.2 \pm 0.9$ & $4.7 \pm 0.7$ & $2.5 \pm 0.5$ & $12.9 \pm_{0.9}^{1.3}$ & $3.5 \pm_{1.1}^{1.2}$ & $1.7 \pm_{0.3}^{0.5}$ & 0.14 \\
\hline 1 & BLAST J084508-433755 & 263.3975 & -0.4588 & $6.5 \pm 0.9$ & $4.5 \pm 0.8$ & $2.0 \pm 0.6$ & $15.7 \pm_{0.3}^{0.4}$ & $1.5 \pm_{0.4}^{0.2}$ & $2.4 \pm_{0.3}^{0.3}$ & 0.14 \\
\hline 2 & BLAST J084509-434544 & 263.5011 & -0.5379 & $4.6 \pm 0.7$ & $3.4 \pm 0.6$ & $1.6 \pm 0.4$ & $12.3 \pm_{1.1}^{1.7}$ & $2.8 \pm_{1.1}^{1.3}$ & $1.1 \pm_{0.2}^{0.4}$ & 0.10 \\
\hline 3 & BLAST J084531-435006 & 263.6001 & -0.5302 & $210.1 \pm 28.1$ & $93.3 \pm 19.6$ & $30.5 \pm 13.3$ & $20.9 \pm_{0.4}^{0.4}$ & $23.2 \pm_{2.5}^{3.2}$ & $206.5 \pm_{14.8}^{11.9}$ & 0.17 \\
\hline 4 & BLAST J084535-435156 & 263.6303 & -0.5414 & $271.9 \pm 30.4$ & $117.7 \pm 18.3$ & $43.2 \pm 10.5$ & $20.9 \pm_{0.4}^{0.4}$ & $28.5 \pm_{3.1}^{3.8}$ & $253.5 \pm_{18.6}^{14.9}$ & 0.18 \\
\hline 5 & BLAST J084538-435507 & 263.6770 & -0.5678 & $13.2 \pm 1.6$ & $7.9 \pm 1.2$ & $4.1 \pm 0.8$ & $13.8 \pm_{0.9}^{1.3}$ & $4.7 \pm_{1.5}^{1.5}$ & $3.5 \pm_{0.7}^{1.0}$ & 0.12 \\
\hline 6 & BLAST J084539-435133 & 263.6334 & -0.5273 & $281.5 \pm 40.9$ & $134.8 \pm 29.5$ & $48.2 \pm 19.9$ & $16.2 \pm_{0.2}^{0.2}$ & $55.9 \pm_{5.3}^{6.8}$ & $106.5 \pm_{7.0}^{5.9}$ & 0.21 \\
\hline 7 & BLAST J084540-430400 & 263.0150 & -0.0316 & $8.0 \pm 1.4$ & $6.6 \pm 1.2$ & $3.7 \pm 0.8$ & $11.1 \pm_{1.1}^{1.6}$ & $8.2 \pm_{3.2}^{4.7}$ & $1.6 \pm_{0.4}^{0.5}$ & 0.15 \\
\hline 8 & BLAST J084542-432721 & 263.3239 & -0.2685 & $7.2 \pm 1.2$ & $5.5 \pm 1.0$ & $3.0 \pm 0.7$ & $11.7 \pm_{1.1}^{1.6}$ & $5.7 \pm_{2.2}^{3.6}$ & $1.5 \pm_{0.3}^{0.4}$ & 0.10 \\
\hline 9 & BLAST J084546-432458 & 263.3005 & -0.2341 & $6.4 \pm 1.0$ & $4.5 \pm 0.8$ & $2.0 \pm 0.6$ & $12.9 \pm_{1.4}^{2.2}$ & $3.1 \pm_{1.3}^{2.2}$ & $1.6 \pm_{0.4}^{0.8}$ & 0.12 \\
\hline 10 & BLAST J084548-435334 & 263.6766 & -0.5269 & $8.0 \pm 2.8$ & $5.1 \pm 2.1$ & $2.4 \pm 1.6$ & $14.2 \pm_{3.4}^{6.4}$ & $2.7 \pm_{1.8}^{6.2}$ & $2.3 \pm_{1.1}^{4.1}$ & 0.12 \\
\hline
\end{tabular}

Notes. Flux densities for BLAST sources are quoted at precisely 250, 350, and $500 \mu \mathrm{m}$ using SED fits to obtain color-corrections for the band-averaged flux densities (Chapin et al. 2008; Truch et al. 2008). The quoted statistical uncertainties are determined from Monte Carlo simulations and do not include calibration uncertainties (see Section 4). The deconvolved or intrinsic source FWHM is obtained from the best-fit source size, $\theta_{\mathrm{fit}}$, and the BLAST beam size, $\theta_{\mathrm{b}}$, as $\theta_{\mathrm{dec}}=\left(\theta_{\mathrm{fit}}^{2}-\theta_{\mathrm{b}}^{2}\right)^{1 / 2}$ (Netterfield et al. 2009).

(This table is available in its entirety in a machine-readable form in the online journal. A portion is shown here for guidance regarding its form and content.)

The catalog of BLAST source positions in Vela-D, including flux densities, is given in Table 1. This list is taken as the reference catalog for all subsequent associations with compact sources detected at all other wavebands described below. The cross-correlation between BLAST sources and objects listed in other catalogs is performed using positional criteria. An archive object is considered to be associated with a BLAST source when their separation is less than the following search radius:

$$
R_{\text {search }}=\left[D_{\text {BLAST }}^{2}+\left(\epsilon_{\mathrm{ptg}}^{\text {archive }}\right)^{2}\right]^{1 / 2},
$$

where $\epsilon_{\mathrm{ptg}}^{\text {archive }}$ is the pointing error associated with the position of the archive source, and we have defined

$$
D_{\mathrm{BLAST}}=\left[\left(D_{\mathrm{dec}} / 2\right)^{2}+\left(\epsilon_{\mathrm{ptg}}^{\mathrm{BLAST}}\right)^{2}+\epsilon_{\mathrm{extr}}^{2}\right]^{1 / 2},
$$

where $D_{\text {dec }}$ is the deconvolved FWHM of the BLAST source. Both $\epsilon_{\mathrm{ptg}}^{\mathrm{BLAST}}$, the BLAST pointing error, and $\epsilon_{\text {extr }}$, the uncertainty in the source position due to the source finding technique, have been estimated to be $\sim 5^{\prime \prime}$.

\subsection{The Millimeter Continuum: SIMBA Data}

Due to the smaller area and lower $\mathrm{S} / \mathrm{N}$ of the SIMBA map (Section 2.2.1), when performing a cross-correlation between the BLAST and SIMBA sources we find a SIMBA counterpart to only 31 BLAST sources, out of 141 in our selected area of Vela-D. We determine the positional offset from each candidate SIMBA counterpart to the nearest BLAST source position and find that the average positional offsets between the BLAST and SIMBA positions are $-3^{\prime \prime} .5$ and -1 .'. 4 in galactic longitude and latitude, respectively, whereas the standard deviations in these two axes are 7".2 and 6."0. The offsets are also randomly distributed, with no particular recognizable pattern, and we attribute them to instrumental pointing and to the different algorithm used for source finding in the BLAST and SIMBA maps.

The cross-correlation between the BLAST and SIMBA catalogs follows the procedure outlined in Section 3.1, assuming
$\epsilon_{\mathrm{ptg}}^{\mathrm{SIMBA}} \simeq 5^{\prime \prime}$ (Massi et al. 2007). We have used the flux densities listed by Massi et al. (2007) for the MMS sources, whereas for the less robust catalog of umms sources (Section 2.2.1) we have performed direct aperture photometry in the SIMBA map. However, if the umms source was found near to another SIMBA source, we retained the original catalog flux density value. In the case that more than one SIMBA source is positionally associated with a single BLAST object, or it is sufficiently close to the BLAST source to affect the photometry, the flux densities of all associated sources are added and used as an upper limit in the BLAST source SED. For those BLAST sources without a SIMBA counterpart (MMS or umms), we estimate an upper limit to the flux density by performing aperture photometry centered over the BLAST coordinates.

\subsection{The MIR and FIR}

\subsubsection{IRAS and Akari}

In Vela-D, we find that several BLAST sources have counterparts in the IRAS Point Source Catalogue version 2.0 (PSC, Helou \& Walker 1988). For the cross-correlation between the IRAS-PSC and the BLAST catalogs we set the positional error $\epsilon_{\mathrm{ptg}}^{\mathrm{IRAS}}$ equal to the semimajor axis of the IRAS error ellipse. Identifications from the IRAS-PSC were found for 26 of the 141 BLAST sources. For sources that lack PSC counterparts or measurements in any of the IRAS bands we produce measurements or upper limits directly from the IRAS Galaxy Atlas maps (IGA; Cao et al. 1997; at 60 and $100 \mu \mathrm{m}$, hereafter IRAS60 and IRAS100). The point spread functions (PSFs) vary across these maps, showing strong elongation along the scan direction. We have used aperture photometry to measure flux densities in the IGA maps. However, the relatively poor angular resolution of IRAS prevents unambiguous determination of the flux in the same volume as was probed by the BLAST sources, due to both extended halo emission and source crowding. Consequently, the IRAS-PSC fluxes are used as upper limits only.

We have found the Akari counterparts associated with all BLAST sources in Vela-D. However, the Akari fluxes have not been used in estimating the best-fit SED (Section 4), although 

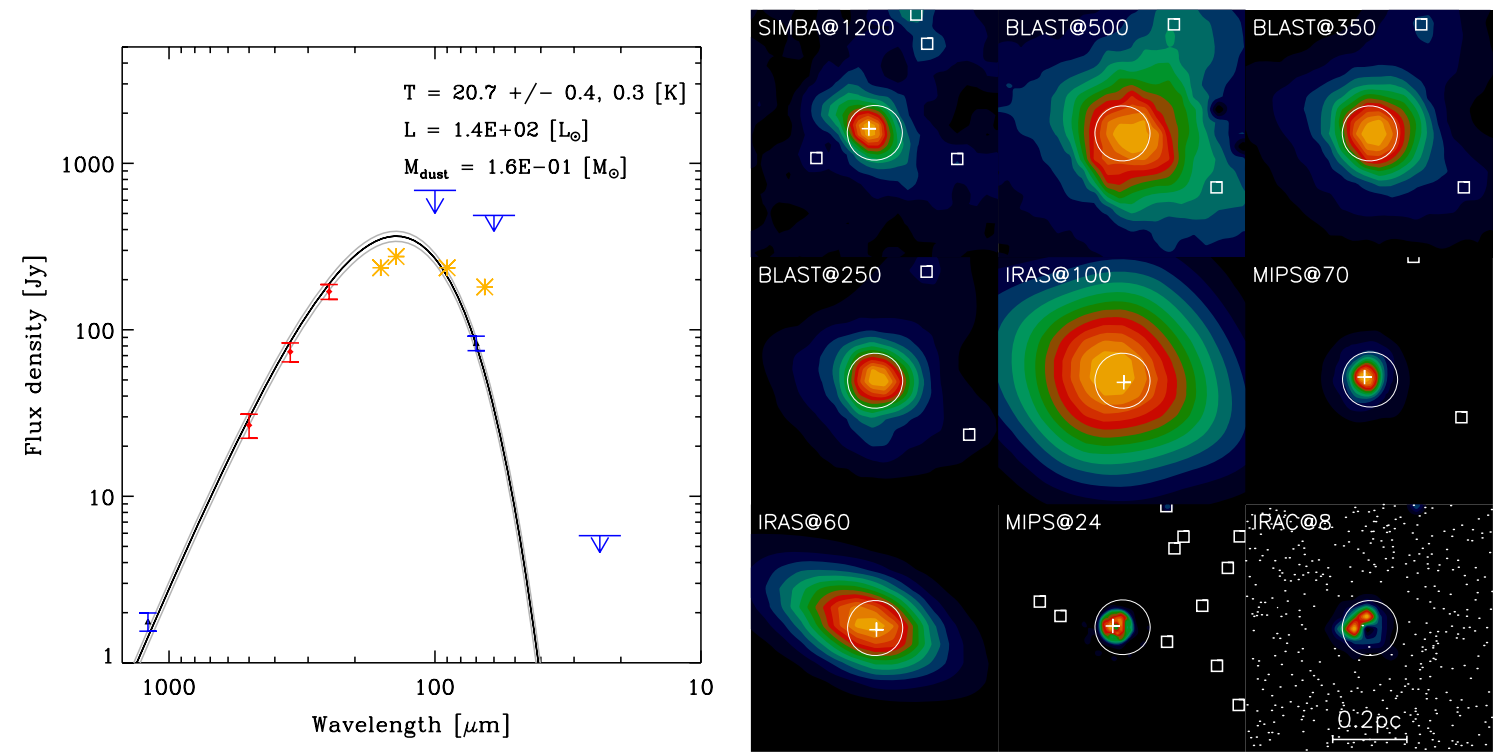

Figure 5. Left: SED of one of the brightest sources in Vela-D, BLAST J084848-433225. Various upper limits are shown and the asterisks represent the Akari fluxes (not used in the fit to the SED). The black line shows the best-fit modified blackbody, at wavelengths $>24 \mu \mathrm{m}$, whereas gray lines show the $68 \%$ confidence envelope of modified blackbody models from Monte Carlo simulations in which the dust emissivity index is fixed at $\beta=2.0$. Right: maps of this source at various wavelengths, from left to right and from top to bottom: SIMBA, BLAST500, BLAST350, BLAST250, IRAS100, MIPS70, IRAS60, MIPS24, and IRAC8. Each map covers a $200^{\prime \prime} \times 200^{\prime \prime}$ region. The large open circle represents the observed FWHM of the BLAST core. The "+" sign marks the position of each specific catalog source (except IRAC) falling within the search radius (Section 3.1) from the nominal position of the BLAST core. In the IRAC map, catalog sources are represented by the white dots. The open squares represent other catalog sources falling outside the search radius.

(A color version of this figure is available in the online journal.)
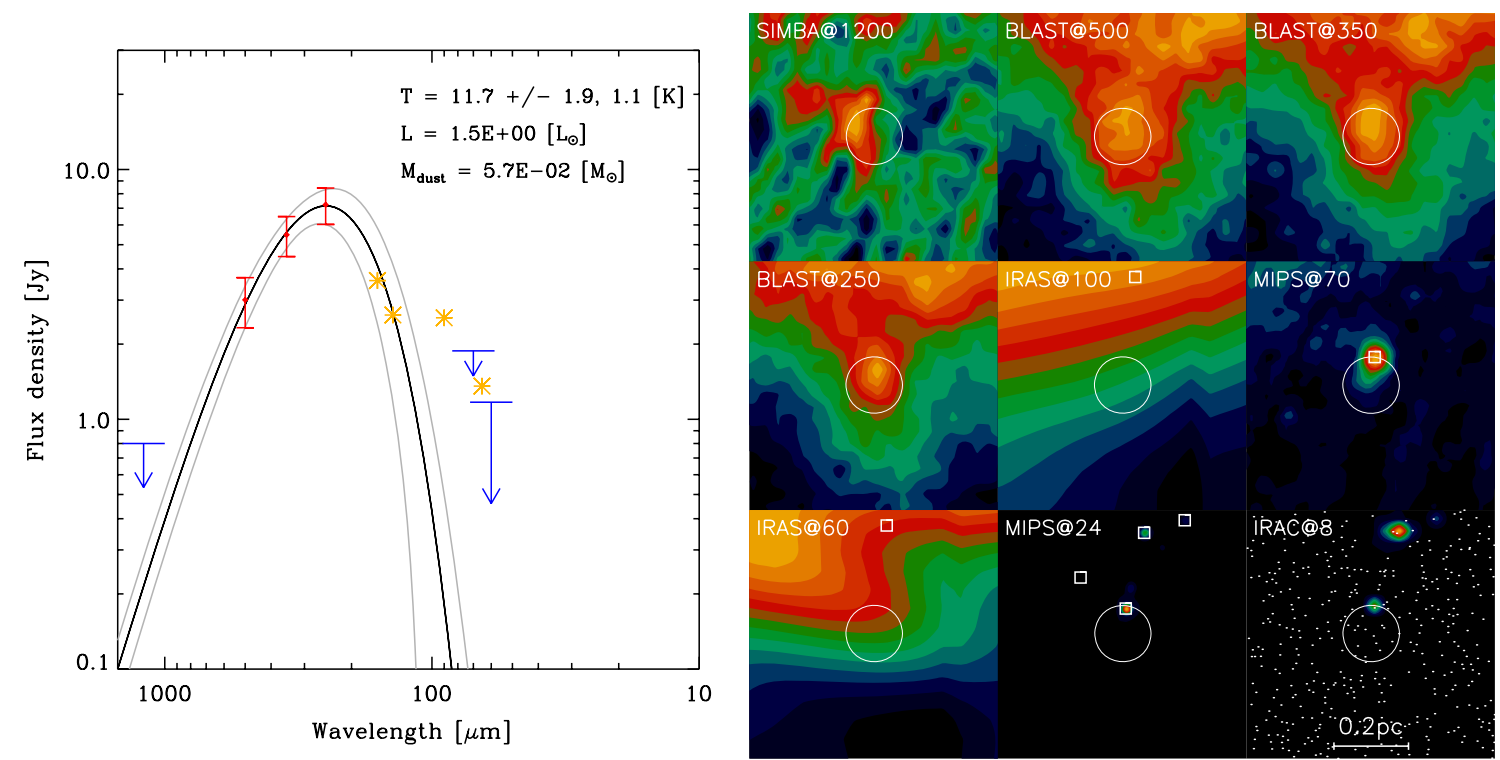

Figure 6. Same as Figure 5 for one of the coldest sources in Vela-D, BLAST J084542-432721.

(A color version of this figure is available in the online journal.)

they are actually shown on the source SED in the two examples presented in Figures 5 and 6. In fact, we have noted that the Akari fluxes from the catalog available to us (which does not include the latest calibration) very often do not quite fall on the best-fit SED determined using all other wavebands. For this reason, we have conservatively determined the SED and the physical parameters of the BLAST sources from the SIMBA, BLAST and MIPS70 fluxes only.

\subsubsection{IRAC and MIPS Photometry}

The IRAC maps were analyzed by means of the DAOPHOT photometric package (see Strafella et al. 2009). Despite the marginal sampling of the PSF by the IRAC cameras, particularly in the $3.5 \mu \mathrm{m}$ and $4.6 \mu \mathrm{m}$ bands, the PSF photometry was preferred because of its better accuracy, with respect to aperture photometry, in dealing with both crowded regions and variable backgrounds. After selection of a sample of bright and unsaturated sources, a point response function ${ }^{23}$ (PRF) was derived in each band and used to fit the sources detected at $\mathrm{S} / \mathrm{N}>5$ to obtain the corresponding photometry (Strafella et al. 2009). For the purpose of cross-correlation between the BLAST and IRAC catalogs, we assumed $\epsilon_{\mathrm{ptg}}^{\mathrm{IRAC}} \simeq 2^{\prime \prime}$.

\footnotetext{
${ }^{23}$ For a comparison between the PRF and PSF, see for example
} http://ssc.spitzer.caltech.edu/irac/psf.html. 
As for IRAC, the Spitzer MIPS observations were exploited to search for possible counterparts to the BLAST sources, also assuming $\epsilon_{\mathrm{ptg}}^{\mathrm{MIPS}} \simeq 2^{\prime \prime}$. Because the MIPS mosaics of the Vela-D cloud are characterized by a particularly strong background level and gradient, we adopted an appropriate spatial filter to smooth all the spatial scales with sizes larger than 20 pixel (corresponding to $50^{\prime \prime}$ and $80^{\prime \prime}$ at 24 and $70 \mu \mathrm{m}$, respectively), preserving the small scale fluctuations. Then, as for IRAC, we performed PSF photometry. This approach allowed us to enlarge the original sample discussed in Giannini et al. (2007).

Because the MIPS70 data lie on the Wien side of the SED, they can strongly constrain the SED (Section 4), and it is thus important to determine whether the integrated emission in each waveband comes from the same volume of material. Submillimeter dust cores, particularly protostellar cores, are likely to have density as well as temperature gradients, implying that the bulk of the emission at different wavelengths may come from substantially different volumes of gas and dust, becoming progressively more optically thick at shorter wavelengths. The power-law density (and, possibly, temperature) profiles of the cores is then coupled with the instrumental response at each waveband, making the exact definition of the "same" core at different wavelengths an imprecise concept at best. However, we note that in a typical protostellar core the wavelength of a photon carrying the mean energy of the SED is $\simeq 50 \mu \mathrm{m}$ and the radius where this photon escapes the cloud is estimated to be $\simeq 13$ AU (e.g., Stahler \& Palla 2005). Thus, we think that the MIPS70 emission can still be mostly associated with the envelope of the protostellar core.

We investigated alternative photometric techniques and, in particular, we compared aperture photometry and a method similar to that used in the BLAST maps (Section 3.1), which may be of relevance to candidate flux-extraction techniques to be used in the forthcoming Herschel multi-wavelength maps. We first convolve the MIPS70 map to the BLAST250 resolution, and then we fitted each compact source in the resulting map with the same Gaussian profile previously extracted from the BLAST maps. However, source crowding may seriously affect the accuracy of aperture photometry, while intrinsically different brightness distributions in the BLAST and MIPS70 wavebands make the extracted flux from fixed-position Gaussians quite unreliable. We thus decided to use catalog values, as determined from PSF photometry, except in three (isolated) sources where the catalog values were clearly inconsistent with the SED constrained by SIMBA and BLAST data points. In these few cases, aperture photometry was used. ${ }^{24}$ For those BLAST sources without a MIPS70 counterpart, an upper limit to the emission was determined also using aperture photometry.

We also checked the MIPS maps for the possible occurrence of detector saturation. In accordance with the MIPS manual ${ }^{25}$ we verified that the elemental integration time, which represents the ultimate threshold to estimate a possible saturation, was short enough that none of the detected sources are saturated at $70 \mu \mathrm{m}$.

\footnotetext{
${ }^{24}$ Following the MIPS manual at http://ssc.spitzer.caltech.edu/mips/apercorr/, we used an aperture radius $R_{\text {apr }}=8.75$ pixel (with 1 pixel $=4^{\prime \prime}$ in the MIPS70 image) and an annulus region delimited by two circles of radii $R_{\text {sky } 1}=9.75$ pixel and $R_{\text {sky } 2}=16.25$ pixel, and an additional correction factor $c=1.308$ $25 \mathrm{http}: / / \mathrm{ssc}$.spitzer.caltech.edu/documents/SOM/
}

\section{MILLIMETER-MIR SEDS}

\subsection{The Approach}

In this section, we describe how, using the SIMBA, BLAST, and MIPS70 photometry (i.e., excluding Akari data points, see Section 3.3.1) discussed in the previous sections, we fit a SED for each BLAST core. However, this is complicated by the possibility that some BLAST cores may be composed by multiple sources. In fact, although BLAST achieved an unprecedented angular resolution at $250 \mu \mathrm{m}$, this is partially compensated by the relatively large distance of Vela-D, thus resulting in a spatial resolution of about $0.1 \mathrm{pc}$. This scale is comparable to the average dense core diameters found by Motte et al. (2007) in Cyg-X and by Rathborne et al. (2008) in the Pipe nebula. The $0.1 \mathrm{pc}$ scale is also comparable with the average diameter of ammonia cores found by Jijina et al. (1999), although it is considerably larger than the diameter found for low-mass cores in nearby SF regions by Ward-Thompson et al. (1999) and Enoch et al. (2008). However, we also find that of the 31 SIMBA sources associated with BLAST cores, only four are actually resolved as a double-core system by the smaller beam of the SEST telescope, suggesting that either the typical scale-length of the cores in Vela-D is considerably smaller than $\sim 0.1 \mathrm{pc}$, or there are relatively few multiple sources.

In addition, we note that for the typical mass of the cores in Vela-D (a few solar masses, see Section 5.3) the power-law relation $M \propto R^{2.6}$ found by Lada et al. (2008) between the core mass and the core radius, would predict (if extrapolated to masses $M \gtrsim 1 M_{\odot}$ ) a core radius of about $0.05 \mathrm{pc}$, well matched with the linear resolution of BLAST at the distance of Vela-D. Furthermore, based on data from Beltrán et al. (2006), who surveyed more massive cores, one can also find that $M \propto R^{p}$ ( $p \simeq 1.6$, Cesaroni 2008, private communication), quite similar to the mass-size relation found by Massi et al. (2007), $M \propto R^{1.7}$, in Vela-D itself. These findings indicate that for core masses larger than about $1 M_{\odot}$ almost all sources have diameters larger than about $0.05-0.1 \mathrm{pc}$.

Nevertheless, even in the BLAST cores that are indeed individual entities, the source would still likely be composed of regions at different temperatures, typically a warmer core (with or without a protostar or protostellar cluster) embedded in a colder and less dense medium (except for those cores possibly heated from an external source). The variations in physical parameters such as temperature and density are likely to be much less critical in starless cores (e.g., André et al. 2000). Our goal is thus to use a simple, single-temperature SED model to fit the sparsely sampled photometry described in Section 3, which will allow us to infer the main physical parameters of each core: mass, temperature, and luminosity. These quantities must be interpreted as a parameterization of a more complex distribution of temperature and density in the core and the equally complex response of each instrument to these physical conditions

\subsection{SED Fitting with Modified Blackbody Function}

Following the method described by Chapin et al. (2008) we assume optically thin emission from an isothermal modified blackbody,

$$
S_{v}=A\left(\frac{v}{v_{0}}\right)^{\beta} B_{v}(T),
$$

where $A$ is a constant, $B_{v}(T)$ is the Planck function, $\beta$ is the dust emissivity index, and the emissivity factor is normalized 
Table 2

$1.2 \mathrm{~mm}-24 \mu \mathrm{m}$ Counterparts

\begin{tabular}{|c|c|c|c|c|c|c|c|c|c|c|c|c|}
\hline Source No. & Source Name & $\begin{array}{c}S_{1200} \\
(\mathrm{Jy})\end{array}$ & $N_{1200}$ & $\begin{array}{l}S_{100} \\
(\mathrm{Jy}) \\
\end{array}$ & $\begin{array}{l}S_{60} \\
(\mathrm{Jy}) \\
\end{array}$ & $N_{\text {IRAS }}$ & $N_{\text {Akari }}$ & $\begin{array}{l}S_{70} \\
(\mathrm{Jy}) \\
\end{array}$ & $N_{70}$ & $\begin{array}{l}S_{24} \\
(\mathrm{Jy}) \\
\end{array}$ & $N_{24}$ & $N_{\mathrm{MSX}}$ \\
\hline 0 & BLAST J084441-431144 & $\cdots$ & $\cdots$ & $3.1^{\mathrm{a}}$ & $0.1^{\mathrm{a}}$ & 0 & 0 & $\cdots$ & $\cdots$ & $\cdots$ & $\cdots$ & 0 \\
\hline 1 & BLAST J084508-433755 & 0.40 & 0 & $12.6^{\mathrm{a}}$ & $1.5^{\mathrm{a}}$ & 0 & 1 & 0.31 & 1 & 0.017 & 1 & 0 \\
\hline 2 & BLAST J084509-434544 & 0.40 & 0 & $\cdots$ & $\cdots$ & 0 & 0 & $\cdots$ & 0 & $\cdots$ & 0 & 0 \\
\hline 3 & BLAST J084531-435006 & 3.17 & 1 & $1688.2^{\mathrm{a}}$ & $620.6^{\mathrm{a}}$ & 0 & 0 & $131.24^{\mathrm{a}}$ & 1 & 1.934 & 1 & 1 \\
\hline 4 & BLAST J084535-435156 & 3.70 & 1 & $1573.0^{\mathrm{a}}$ & $592.0^{\mathrm{a}}$ & 0 & 2 & $161.44^{\mathrm{a}}$ & 1 & 1.280 & 2 & 0 \\
\hline 5 & BLAST J084538-435507 & 0.40 & 0 & $123.8^{\mathrm{a}}$ & $1.3^{\mathrm{a}}$ & 0 & 0 & $5.77^{\mathrm{a}}$ & 0 & 0.194 & 1 & 0 \\
\hline 6 & BLAST J084539-435133 & 3.59 & 1 & $1519.9^{a}$ & $685.5^{\mathrm{a}}$ & 0 & 0 & 16.97 & 1 & $\cdots$ & 0 & 1 \\
\hline 7 & BLAST J084540-430400 & $\cdots$ & $\cdots$ & $\cdots$ & $\cdots$ & 0 & 0 & $\cdots$ & $\cdots$ & $\cdots$ & $\cdots$ & 0 \\
\hline 8 & BLAST J084542-432721 & 0.40 & 0 & $\ldots$ & $0.1^{\mathrm{a}}$ & 0 & 1 & $1.30^{\mathrm{a}}$ & 0 & $\ldots$ & 0 & 0 \\
\hline 9 & BLAST J084546-432458 & 0.40 & 0 & $13.4^{\mathrm{a}}$ & $0.9^{\mathrm{a}}$ & 0 & 0 & $0.65^{\mathrm{a}}$ & 0 & $\cdots$ & 0 & 0 \\
\hline 10 & BLAST J084548-435334 & 0.40 & 0 & $262.5^{\mathrm{a}}$ & $23.7^{\mathrm{a}}$ & 0 & 0 & $5.00^{\mathrm{a}}$ & 0 & 0.057 & 1 & 0 \\
\hline
\end{tabular}

Notes. Flux density $(S)$ and number $(N)$ of sources associated with BLAST objects for each catalog considered: SIMBA, IRAS, Akari, MIPS70, MIPS24, and $M S X$. Fluxes are catalog fluxes, unless noted otherwise. When $N=0$, but the flux density $S$ is not zero, then an upper limit to the flux is listed, either estimated by aperture photometry (indicated by "a") or by determining the noise in the map. The search radius is a variable function of both BLAST and the ancillary instrument positional uncertainties (see Section 3.1).

a Aperture photometry has been used.

(This table is available in its entirety in a machine-readable form in the online journal. A portion is shown here for guidance regarding its form and content.)

at a fixed frequency $\nu_{0}$. We then write the factor $A$ in terms of a total (gas + dust) core mass, $M$, the dust mass absorption coefficient $\kappa_{0}$ (evaluated at $v_{0}$ ), and the distance to the object, $d$ (which is here taken as $700 \mathrm{pc}$ for all cores):

$$
A=\frac{M \kappa_{0}}{R_{\mathrm{gd}} d^{2}} .
$$

Since $\kappa_{0}$ refers to a dust mass, the gas-to-dust mass ratio, $R_{\mathrm{gd}}$, is required in the denominator to infer total masses. We adopt $\kappa_{0}=16 \mathrm{~cm}^{2} \mathrm{~g}^{-1}$, evaluated at $\nu_{0}=c / 250 \mu \mathrm{m}$. While the actual value of $R_{\mathrm{gd}}$ may depend on the physical conditions of the local ISM or molecular cloud (e.g., Frisch \& Slavin 2003, Vuong et al. 2003, and references therein) we adopt the value of $R_{\mathrm{gd}} \simeq 100$ for simplicity. Equation (3) is fitted to all of the BLAST and archive photometry, from 1200 to $70 \mu \mathrm{m}$ using $\chi^{2}$ optimization. Color correction of the BLAST flux densities, before comparing them with other photometry, is described in Chapin et al. (2008) and Truch et al. (2008), and the resulting BLAST color-corrected fluxes are listed in Table 1. $\beta$ is not well constrained and we choose to fix it to $\beta=2.0$ (see Netterfield et al. 2009 for a discussion about the adopted values for $\kappa_{0}$ and $\beta$ ), while only $A$ and $T$ are allowed to vary.

"Survival analysis" is adopted to properly include the upperlimits in the calculation of $\chi^{2}$ (see the discussion in Chapin et al. 2008), and we choose to use all photometry at wavelengths shortward of $70 \mu \mathrm{m}$ as upper-limits. This is motivated by the following reasons: (1) emission at $24 \mu \mathrm{m}$ and shorter is coming from volumes of material quite different from those emitting at longer wavelengths, which is typically associated with the coldest and less dense dust; (2) at shorter wavelengths there can be significant contributions from hotter dust grains, PAH bands and other spectral features; and, (3) in protostellar regions multiple sources may be present that are simultaneously associated with the emission in the FIR/submillimeter wavebands. In the latter case, these multiple fluxes are all summed together and taken as upper-limits in the SEDs.

Figure 5 shows an example SED for the source BLAST J084848-433225, one of the brightest BLAST objects in the sample, with a best fit temperature to the mm-FIR data of $20.7 \mathrm{~K}$.
Likewise, Figure 6 shows the SED of BLAST J084542-432721, one of the coldest objects in Vela-D $(T=11.7 \mathrm{~K})$. As seen in this example, observations across the BLAST wavelength range clearly reveal a turnover in the FIR SED for the coldest objects. The thumbnails shown in the bottom panel of Figure 6 clearly emphasize the lack of counterparts in the shorter-wavelength bands for this object, though it is located near a warmer object with clear MIPS24 and MIPS70 counterparts.

Particularly for the coldest objects, undetected in the FIR-MIR wavebands, there is a strong degeneracy between the values of $\beta$ and $T$, i.e., there can be a large spread in the values of these parameters that can fit the SED equally well. This effect is less important for more evolved sources, which usually have a FIR-MIR counterpart, thus better constraining the SED shape and location of the FIR peak. Fixing the value of $\beta=2.0$ has the advantage of reducing the inferred errors in $T$ (Chapin et al. 2008) and makes the shape of the SED depend on temperature only, but it clearly prevents the analysis of possible variations of $\beta$ as a function of other source parameters. The physical parameters derived from the best-fit SED for each source are listed in Table 1, whereas the flux densities used in the SED and the counterparts found in each catalog are listed in Table 2.

\section{COMPARING THE PHYSICAL PROPERTIES OF STARLESS AND PROTOSTELLAR CORES}

\subsection{Separating Starless and Protostellar Cores}

In this section, we consider the properties of starless and protostellar cores. The former will be defined as those cores without an internal source of luminosity (i.e., a protostar), and may be further defined as "pre-stellar" when they appear to be gravitationally bound. Protostellar cores, on the other hand, have an embedded self-luminous source (which may also be a cluster of objects) and possibly show other signs of SF, such as molecular outflows.

Starless and protostellar cores may be differentiated by identifying MIR counterparts associated with the submillimeter cores. Here, we define as protostellar those cores which have a MIPS24 compact source, with flux greater than $3 \mathrm{mJy}$ (see 
Section 5.2), within the search radius defined in Section 3. For the BLAST cores outside the area covered by the MIPS24 map (a total of 52 objects), we use the association with compact sources from the Midcourse Space Experiment (MSX) catalog, although the sensitivity limit is much higher than that of the MIPS24 catalog (e.g., Egan et al. 1999), and thus some of the starless cores outside the MIPS covered area might have a yet undetected MIR counterpart (see Sections 5.3.1, 5.3.2, and 5.4 for a discussion of this point). We note that some of the point-like objects detected by MIPS24 may have a very low luminosity, so we would expect that they neither heat up the core overall nor increase its luminosity. Thus, the emission of these early protostellar cores in the BLAST wavebands will look much the same as the starless cores.

As SF in the core proceeds, more and more luminosity is generated, mostly from accretion but also from the quasi-static contraction of the interior and, later, from nuclear fusion. This luminosity is re-processed by dust and hence these objects will appear more luminous and warmer (and thus also with a larger luminosity-to-mass ratio, $L / M$ ). In terms of the observational properties, one should therefore expect a smooth transition from the pre- to the protostellar phase. Our criteria are not meant to provide a sharp separation between these two classes of objects, but should provide guidelines for understanding the physical conditions where the transition takes place.

\subsection{MIPS24 and IRAC Counterparts}

Analyzing in more detail the association of MIPS24 pointsources with the BLAST cores, we find that only two cores, BLAST J084612-432337 and BLAST J084745-432637, have MIPS24 counterparts with fluxes lower than $3 \mathrm{mJy}$, i.e., the threshold selected by Enoch et al. (2008) to eliminate most extragalactic interlopers. We thus classify these two cores as starless. By comparing the surface density of MIPS24 catalog sources in the area covered by the MIPS24 data with a circular area of radius equal to the search radius, we estimate the probability of finding a chance association with a MIPS24 source, brighter than $3 \mathrm{mJy}$, within the search radius to be less than $10 \%$. Thus, although chance associations of BLAST cores with MIPS24 point sources seem unlikely, in Section 5.4 we analyze how these might potentially affect the overall distribution of physical parameters between starless and protostellar objects.

We also find cases (a total of 26 objects), where a source is detected in the MIPS24 band, but not in the MIPS70 band. As an example, we show in Figure 7(c) source BLAST J084842431735, where we find an associated point source from the MIPS24 catalog, but no point or even compact MIPS70 emission at the position of the BLAST core. In more than one third of other similar cases, some compact weak or halo emission associated with the BLAST core is found in the MIPS70 map. An example is shown in Figure 7(d): we can clearly see a weak compact source of emission in the MIPS70 map at the position of the BLAST core, though no point source is found in the catalog. In such cases, it is likely that a combination of intrinsically low $70 \mu \mathrm{m}$ emission and/or instrumental artifacts (e.g., stripes) in the MIPS70 maps may mask the $70 \mu \mathrm{m}$ counterpart to the MIPS24 source associated with the BLAST core. Geometrical projection effects may also be playing an important role at wavelengths $\lesssim 100 \mu \mathrm{m}$ for sources with an embedded protostar (e.g., Whitney et al. 2004).

Identifying the NIR/MIR young protostellar counterparts associated to the BLAST cores is beyond the scope of this work. However, we have carried out a preliminary search of IR candidates among the IRAC sources, selected using the following criteria: (1) spectral index $\alpha_{\mathrm{sp}}=d \log \left(\lambda S_{\lambda}\right) / d \log (\lambda)$, estimated using 2MASS, IRAC, and MIPS24 data, with values $\alpha_{\mathrm{sp}}>0.3$; (2) the source must be detected in the IRAC $8 \mu \mathrm{m}$ band; and (3) when no MIPS24 flux is available, the flux extrapolated from 2MASS and IRAC data must be consistent with an upper limit in the MIPS24 band. With these criteria, we find that $69 \%$ of the starless cores, as defined above, within the area in Vela-D covered by IRAC do not have an IRAC candidate. Likewise, we find that $73 \%$ of the protostellar cores do have an IRAC candidate. Other criteria can be found in the literature (see, e.g., De Luca et al. 2007, Enoch et al. 2009), but our quick search indicates that the starless and protostellar populations have been fairly accurately characterized.

However, we can also use other signposts of early SF activity, such as protostellar jets and molecular outflows. In fact, we have identified 13 BLAST cores associated with the $\mathrm{H}_{2}$ jets and knots observed by Giannini et al. (2005), Giannini et al. (2007) and De Luca et al. (2007), and all of them had been correctly identified as protostellar, except the core BLAST J084822-433152, which has no MIPS24 (and no MIPS70) counterpart and had thus been classified as starless. However, the association of this specific core with an $\mathrm{H}_{2}$ jet is not as robust as the others (De Luca et al. 2007) and thus given its other properties we have decided to keep its classification as starless. We have also identified the BLAST cores associated with the ${ }^{12} \mathrm{CO}(1-0)$ molecular outflows observed by Elia et al. (2007) and found that only one core, BLAST J084805-435415, had been previously incorrectly classified as starless.

As an additional category, we also find some BLAST cores that are detected in MIPS24, MIPS70 (and in the IRAC bands) that are characterized by quite low temperatures. An example is source BLAST J084928-440426, shown in Figure 7(e), which is clearly associated with bright MIPS24 and 70 point sources. However, its temperature, as determined from the best fit to the SED, is only $13.1 \mathrm{~K}$. Such a cold source is not expected to emit a detectable flux in the MIPS70 band and we are therefore left with two possible scenarios: (1) either the $70 \mu \mathrm{m}$ flux is mostly emitted by the warmer central source associated with the BLAST core, and thus the BLAST fluxes represent the contribution from a much colder envelope; or, (2) the BLAST fluxes and the MIR emission are associated with two (or more) separate objects, a cold, starless core detected by BLAST, and a nearby proto-stellar core detected by MIPS with negligible emission in the submillimeter. Because of our inability to discriminate between these two scenarios, and for consistency with the criteria given above, we consider these cores as protostellar.

Finally, we note that in objects such as core BLAST J084928440426 (of the previous example) although associated with point sources in the IRAC and MIPS wavebands, the peak of the submillimeter emission corresponds to a minimum in the MIPS24 and IRAC8 maps, as shown in Figure 7(e). This and other similar cores stand out as dark shadows against the diffuse 8 and $24 \mu \mathrm{m}$ emission.

\subsection{Distribution of Physical Parameters \\ 5.3.1. Best-fit Values: Temperature}

Temperature distributions for starless and protostellar cores in Vela-D are shown in Figure 8. The histograms refer to the values of the physical parameters as obtained from the best fit to the SED of each source found in the area defined in Section 2.1 


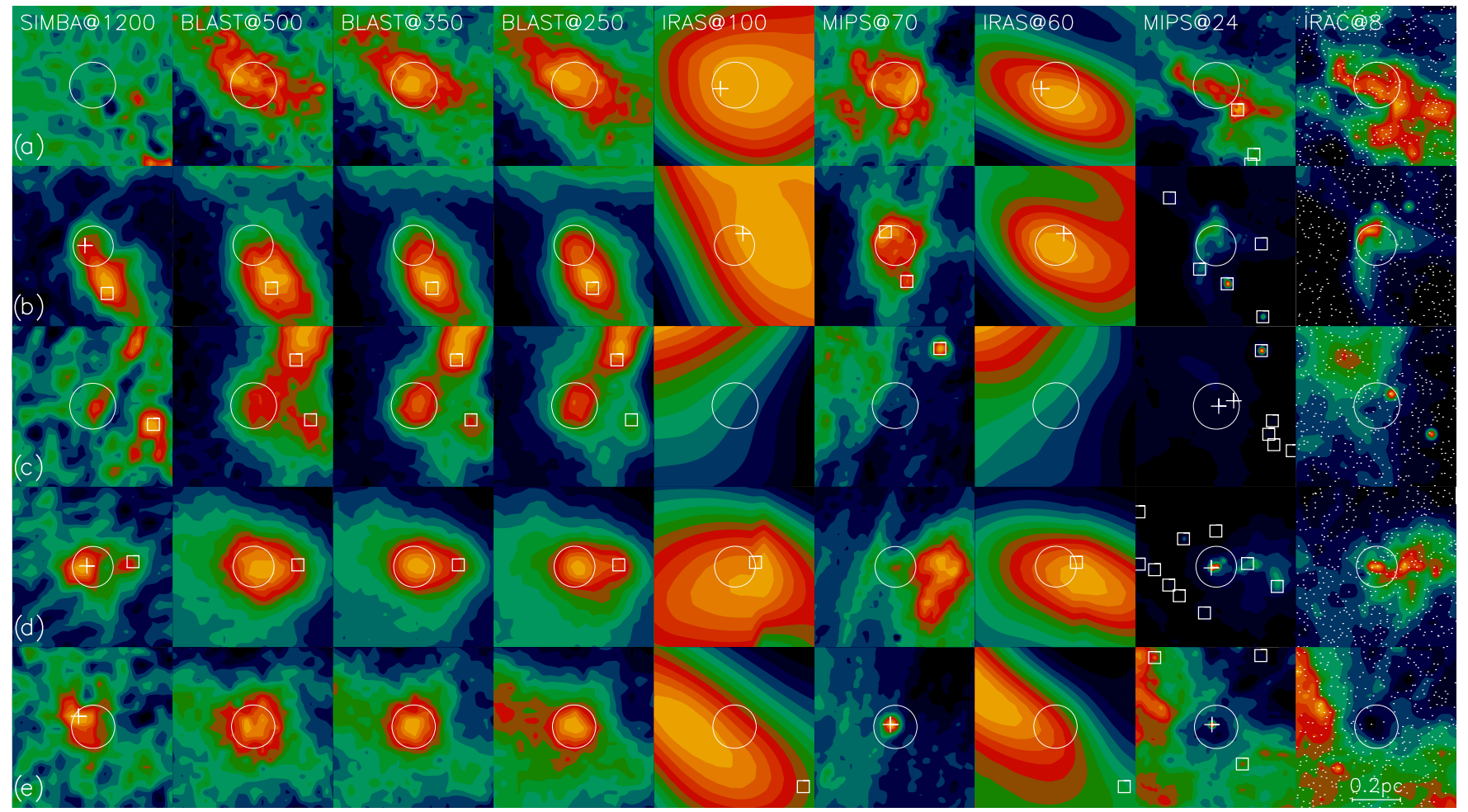

Figure 7. Rows (a) and (b) show two examples of cores (BLAST J084805-435415 and BLAST J084902-433802, respectively) with no MIPS24 point source counterpart, but with MIPS70 compact emission. Core BLAST J084805-435415 is indeed protostellar as it is found to be associated with a molecular outflow (see the text). Rows (c) and (d) show two cores (BLAST J084842-431735 and BLAST J085010-431704, respectively) with no compact MIPS70 emission but with MIPS24 counterparts. Row (e) represents an example of a cold BLAST core (BLAST J084928-440426) with both MIPS24 and MIPS70 counterparts. Wavebands are ordered from left to right as follows: SIMBA, BLAST500, BLAST350, BLAST250, IRAS100, MIPS70, IRAS60, MIPS24, and IRAC8. Symbols have the same meaning as in Figure 5.

(A color version of this figure is available in the online journal.)

and Figure 1. The effects, on the overall distribution, of the errors on the parameters determined for each individual source will be discussed in Section 5.3.3. It must also be noted that only 89 objects are located within the common area covered by the MIPS24 and MIPS70 maps (see Figure 3).

In Figure 8, we note that the temperature distribution has a wide peak at $T \sim 15 \mathrm{~K}$, with a sharp cutoff at the low end around $11-12 \mathrm{~K}$ and also at the high end, at about $18-20 \mathrm{~K}$. In fact, less than $7 \%$ of the sources are warmer than $20 \mathrm{~K}$, and they are mostly protostellar objects. The median value for the whole sample is $15.4 \mathrm{~K}$, and we note that protostellar cores are slightly warmer (median temperature $15.7 \mathrm{~K}$ ) than starless cores (median temperature $14.6 \mathrm{~K}$ ). However, if we restrict ourselves to mass ranges for which we are progressively more complete, then the temperature difference between starless and protostellar cores appears to become increasingly more significant, as shown in Table 3 and by the dashed lines in the top-left panel of Figure 8 . Clearly, in this case the sample size also becomes smaller. The higher value of the overall median temperature, as compared for example to the Pipe cores (Rathborne et al. 2008) or the cores in Vela-C, suggests that the cores in Vela-D are indeed in a later stage of evolution. The range of values found for the spectral index (Section 5.2) is an evidence for different evolutionary stages within the protostellar population. However, we have not attempted to separate the starless and protostellar populations into further sub-groups.

One question of interest is whether temperature is a good discriminator of starless and protostellar cores, in a statistical sense. The overlap between the temperature distributions of starless and protostellar cores in Figure 8, besides being affected by completeness effects (see Table 3), is also likely to be a consequence of the smooth transition from the pre- to the protostellar phase, as discussed in Section 5.1. This can also be seen in the left panel of Figure 9, where we show the fraction (out of the total number of cores in each bin) of starless and protostellar cores, as a function of core temperature, in the whole Vela-D region. The plot clearly shows that the fractions of starless and protostellar cores have opposite trends with temperature. This trend is also visible, though to a lesser extent, in the smaller region covered by the MIPS24 map (right panel of Figure 9), where we are able to better discriminate starless from protostellar cores (Section 5.1). If sensitive MIR observations were also available outside the MIPS24 map one would expect the left panel of Figure 9 to become more like the histogram in the right panel. Possible bias effects caused by the use of MIPS24 and MSX data in different regions of Vela-D are later discussed in Section 5.4.

The distribution of temperature among the Vela-D sources can also be used to search for evidence of evolution within the protostellar cores found in the MIPS24 map. Thus, in Figure 10 we plot the MIPS24 flux as a function of core temperature, and we also separate cores in two different mass ranges, to be able to partially disentangle evolutionary effects from core mass-related effects.

Figure 10 then shows two distinct features: (1) first, there is a trend for the MIPS24 flux to increase with temperature; and, (2) this trend is visible also within the two selected mass ranges, though these two subsets appear to populate different regions of 

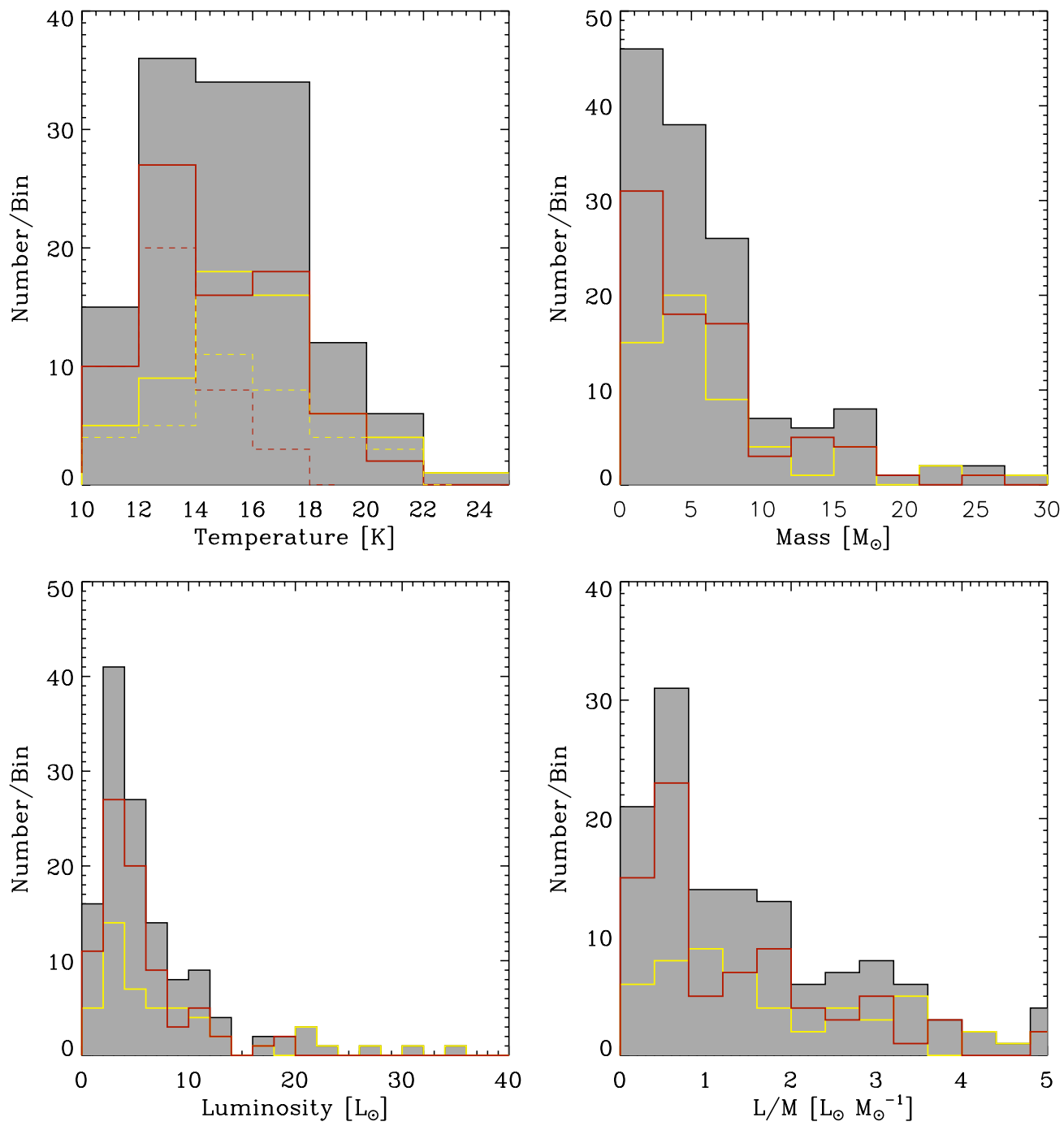

Figure 8. Overall distributions of physical parameters of the BLAST cores in the Vela-D region shown in Figure 1 and defined in Section 5.3.1 and Section 5.3.2 (shaded histograms). Protostellar (yellow solid lines) and starless cores (red solid lines) are defined in the text. Shown are the distribution of the temperatures (top-left panel), which also includes the distributions for cores with $M>4.2 M_{\odot}$ (dashed lines; see Table 3), masses (top-right panel), luminosities (bottom-left panel) and luminosity-to-mass ratios (bottom-right panel).

(A color version of this figure is available in the online journal.)
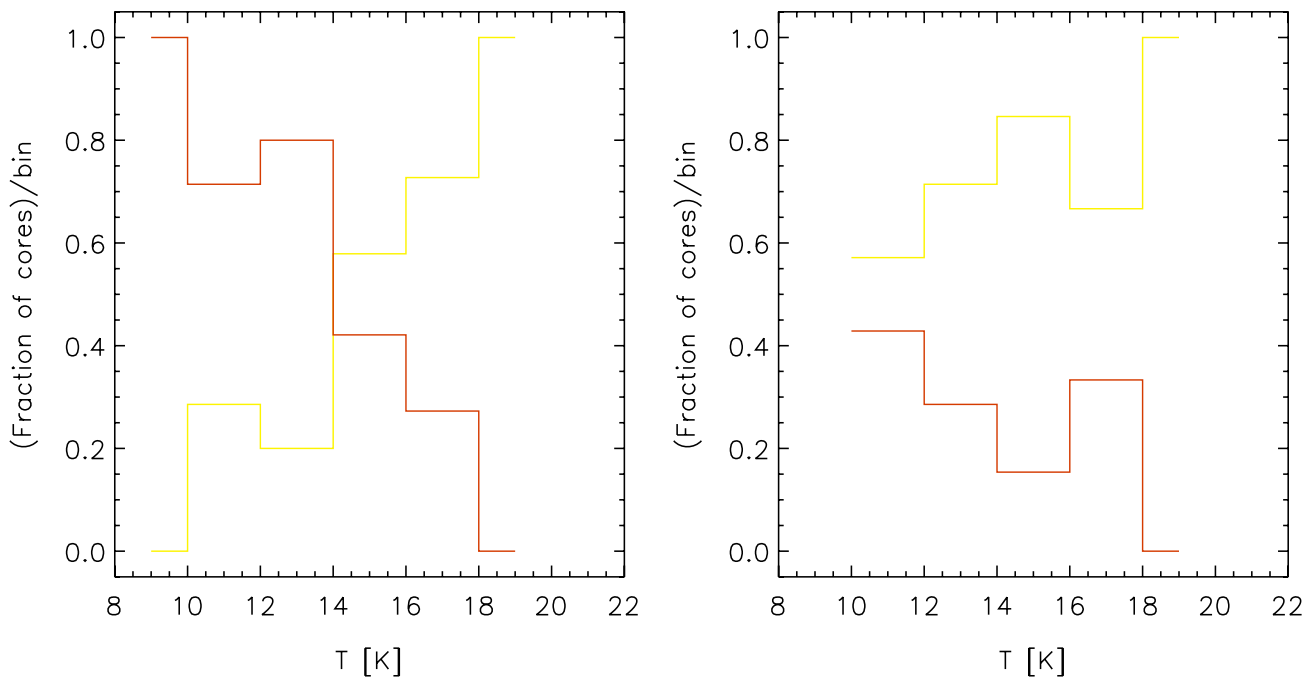

Figure 9. Left: fraction of starless (red line) and protostellar (yellow line) cores vs. temperature, in the whole Vela-D region and only for cores with $M>4.2 M_{\odot}$. Right: same as above in the region covered by the MIPS24 map (see discussion in Section 5.3.1 and Section 5.4).

(A color version of this figure is available in the online journal.) 
Table 3

Median Values of Main Physical Parameters of Starless and Protostellar Cores in Vela-D

\begin{tabular}{lcrcccccc}
\hline \hline \multirow{2}{*}{ Physical Parameter } & \multicolumn{3}{c}{ Starless } & & \multicolumn{3}{c}{ Protostellar } \\
\cline { 3 - 5 } \cline { 8 - 9 } & & All & $M>4.2 M_{\odot}$ & $M>11 M_{\odot}$ & & All & $M>4.2 M_{\odot}$ & $M>11 M_{\odot}$ \\
\hline$T$ & $(\mathrm{~K})$ & 14.6 & 13.1 & 12.9 & & 15.7 & 15.5 & 17.2 \\
$M$ & $\left(M_{\odot}\right)$ & 4.6 & 7.0 & 15.3 & & 4.8 & 8.7 & 16.5 \\
$L_{\mathrm{FIR}}$ & $\left(L_{\odot}\right)$ & 4.4 & 4.5 & 7.0 & & 7.5 & 10.9 & 84.7 \\
$L_{\mathrm{FIR}} / M$ & $\left(L_{\odot} M_{\odot}^{-1}\right)$ & 1.0 & 0.5 & 0.5 & & 1.6 & 1.5 & 2.7 \\
\hline
\end{tabular}

Note. Median values of the best fits to the SEDs. The two completeness limits, $M>4.2 M_{\odot}$ and $M>11 M_{\odot}$, correspond to sources with $T>12 \mathrm{~K}$ and $T>10 \mathrm{~K}$, respectively.

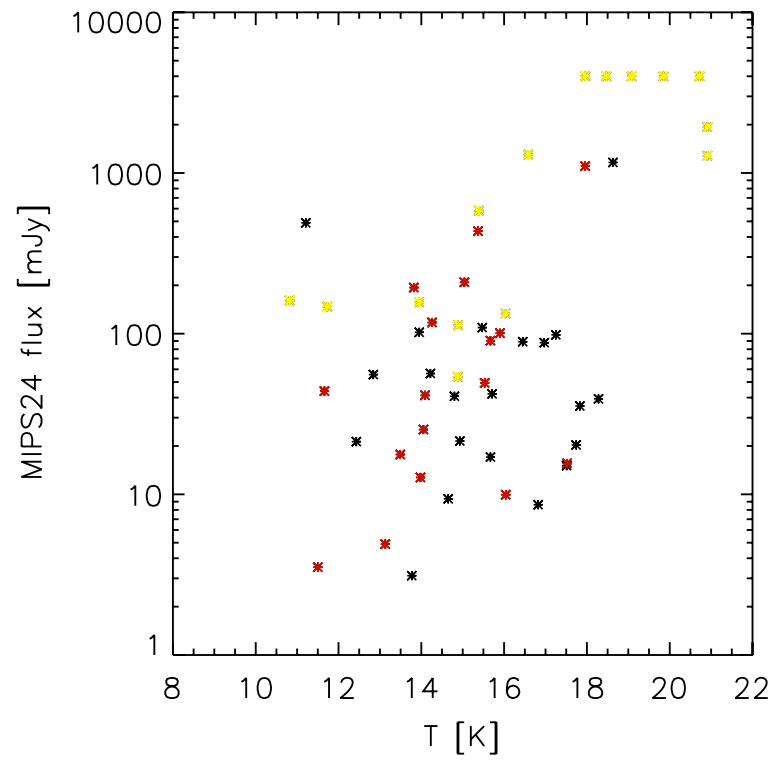

Figure 10. MIPS24 flux of the protostellar cores vs. core temperature. The red and yellow symbols represent the complete sample of cores, with mass in the ranges $4.2<M<9 M_{\odot}$, and $M>9 M_{\odot}$, respectively. The black symbols represent cores with masses $<4.2 M_{\odot}$. Sources with flux $\geqslant 4000 \mathrm{mJy}$ are saturated (Giannini et al. 2007; Strafella et al. 2009).

(A color version of this figure is available in the online journal.)

the plot. The sources with higher masses, in fact, appear to also have somewhat higher MIPS24 fluxes and temperatures. We tentatively explain the main trend of flux versus temperature as an evidence of different evolutionary phases within the population of proto-stellar cores, while the partial segregation of the two subsets described above appears to be associated with the mass of the core. This result is consistent with the trend in the $L_{\text {bol }}-T_{\text {bol }}$ diagram observed by Enoch et al. (2009) and modeled by Young \& Evans (2005; see also Whitney et al. 2003). The increase in $L_{\mathrm{bol}}$ is accompanied by a general increase of the flux density at MIR wavelengths.

\subsubsection{Best-fit Values: Mass and Luminosity}

The overall mass distribution in Figure 8 shows, as expected, an almost monotonic decrease from lower to higher masses. It also shows very few cores with $M>10 M_{\odot}$. In fact, the median value for the whole sample is $4.7 M_{\odot}$, whereas from Table 3 we note that the median masses of starless and protostellar cores are not significantly different, suggesting that the envelope mass is not severely affected by the transition between the two phases.

In Figure 8, we also show the FIR luminosity distribution of the cores in Vela-D. FIR luminosities, $L_{\mathrm{FIR}}$, are the integrated luminosities from the modified blackbody fit. Though not shown (to make the histogram easier to read), we find 11 sources $(\simeq 8 \%$ of the total, all protostellar but one, BLAST J084539-435133) with luminosities higher than $40 L_{\odot}$. The luminosity histogram shows a strong peak at $L_{\text {FIR }} \sim 4 L_{\odot}$ and a rapidly decreasing trend at higher luminosities. The median value for the whole sample is $4.6 L_{\odot}$ and contrary to the mass distribution, we find a significant difference between the median luminosity of starless and protostellar cores (see Table 3).

Although the luminosity-to-mass ratio, $L_{\mathrm{FIR}} / M$, is essentially equivalent to temperature (see later Figure 11), it is useful to show it separately in Figure 8 and in Table 3, as well as in the rest of the paper. Like temperature, this ratio is also expected to increase with time as more and more gas is converted into stars during the SF process and the embedded protostar, or protocluster, becomes more luminous. For the simple SED model adopted, we find a range $L_{\mathrm{FIR}} / M \sim 0.2-40 L_{\odot} M_{\odot}^{-1}$, with $\simeq 10 \%$ and $\simeq 3 \%$ of the sources having $L_{\mathrm{FIR}} / M>5 L_{\odot} M_{\odot}^{-1}$ and $L_{\mathrm{FIR}} / M>10 L_{\odot} M_{\odot}^{-1}$, respectively. The median value for the whole sample is $1.4 L_{\odot} M_{\odot}^{-1}$. Like temperature, the higher value of the luminosity-to-mass ratio found in protostellar cores does suggest that they are indeed at a later stage of evolution.

The temperatures and masses of the cores are also shown in Figure 1, for both starless and protostellar cores, where we note some interesting features. First, one can see the higher concentration of protostellar cores toward the central filaments. While this might partly be a consequence of being unable to identify all protostellar cores outside the MIPS24 area, we think that the presence here of many IRS sources (Section 2.3) would indeed suggest that this region of Vela-D is in a later stage of evolution. We also tentatively note that the most massive cores are rarely found in isolation (such as several of the IRS sources); instead, they are often associated with small clusters of objects. On the other hand, the most isolated cores tend to be low-mass objects. These characteristics, though no quantitative measurement is involved, seem to be in agreement with more systematic observations (e.g., Faustini et al. 2009) and numerical simulations (e.g., Smith et al. 2009). Another interesting feature is the filament at $l \sim 262.80$ and $b \sim+0.3$ which is exclusively composed of massive and cold starless cores.

Finally, in Figure 11 we show a plot of the luminosity as a function of mass. The plot shows a high concentration of points at temperatures $\lesssim 15 \mathrm{~K}$, or $L_{\mathrm{FIR}} / M \lesssim 0.65 L_{\odot} M_{\odot}^{-1}$, which clearly reproduces the results already shown in Figure 8. Separated from the main concentration of sources, we note in Figure 11 a small group of nine bright objects with flux density (at $250 \mu \mathrm{m}$ ) $>100 \mathrm{Jy}$ and $L_{\mathrm{FIR}}>80 L_{\odot}$. In fact, five of these correspond to known IRS sources (Liseau et al. 1992), IRS17 to IRS21, and all of these objects belong to small clusters of sources (Section 2.3). 

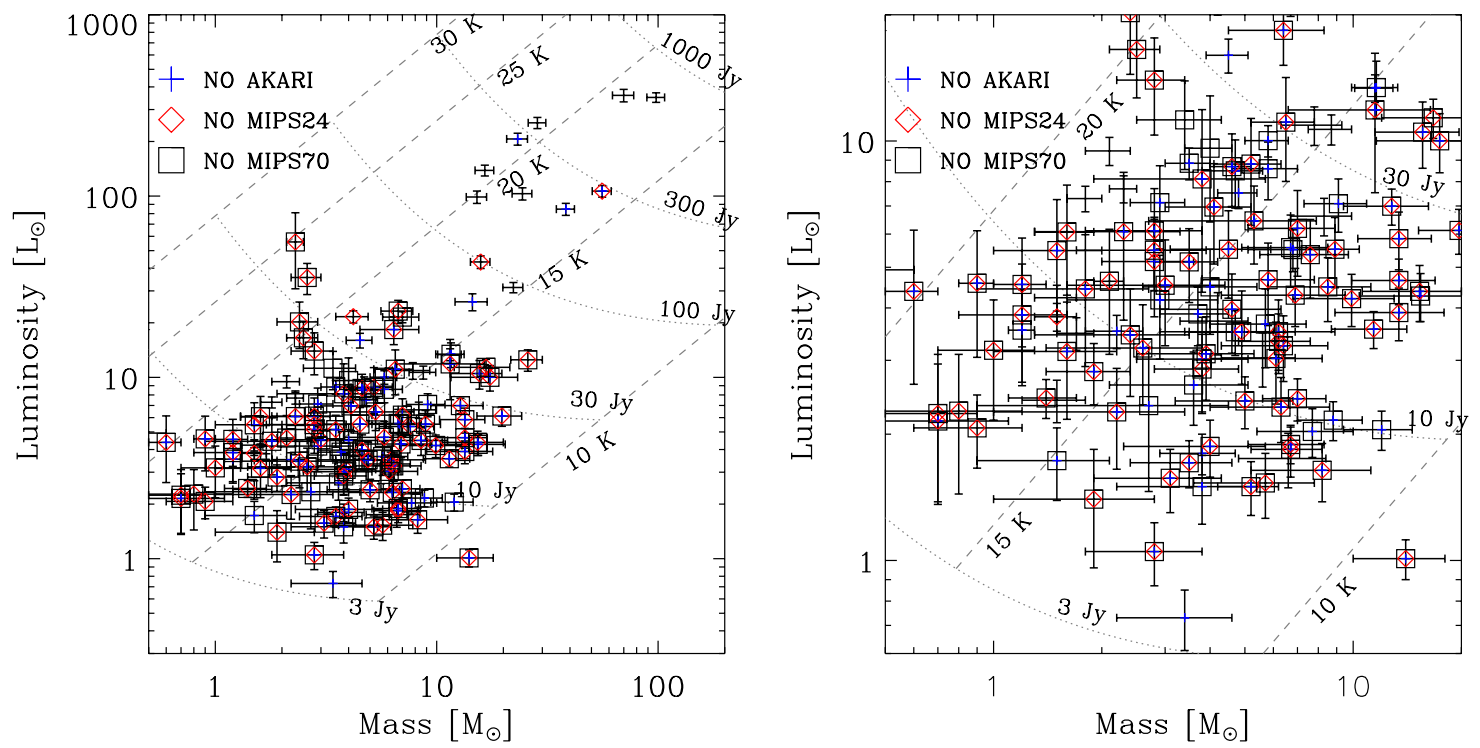

Figure 11. Left: luminosity vs. mass for the BLAST sources in Vela-D. The errors shown in mass and luminosity are estimated from the range of temperatures consistent with the BLAST and archival photometric data under the assumption of the simple SED model of Equation (3). We do not include additional sources of error involving the distance to Vela-D and the adopted values of $\beta, \kappa_{0}$, and gas-to-dust mass ratio. The dashed lines are loci at constant $T=10,15,20,25$, and $30 \mathrm{~K}$, corresponding (assuming a modified blackbody SED with $\beta=2.0$ ) to constant $L_{\mathrm{FIR}} / M$ values of $0.07,0.65,3.2,10.9$ and $29.6 L_{\odot} M_{\odot}^{-1}$ Roughly orthogonal to these are loci (dotted lines) of constant $250 \mu \mathrm{m}$ flux density, ranging from 3 to $1000 \mathrm{Jy}$, using the same model. Right: zoomed plot at low masses and luminosities.

(A color version of this figure is available in the online journal.)

Table 4

Average Values from Monte Carlo Simulations for All Cores in Vela-D

\begin{tabular}{lcrrrrr}
\hline \hline \multirow{2}{*}{ Physical Parameter } & \multicolumn{2}{c}{ Starless } & & \multicolumn{2}{c}{ Proto-stellar } \\
\cline { 3 - 4 } & & mm-MIR & \multicolumn{1}{c}{ BLAST } & & mm-MIR & \multicolumn{1}{c}{ BLAST } \\
\hline$T$ & $(\mathrm{~K})$ & $13.1 \pm 0.3$ & $13.1 \pm 0.3$ & & $15.6 \pm 0.2$ & $15.7 \pm 0.5$ \\
$M$ & $\left(M_{\odot}\right)$ & $8.5 \pm 0.8$ & $8.7 \pm 0.9$ & & $9.1 \pm 0.8$ & $9.7 \pm 1.1$ \\
$L_{\mathrm{FIR}}$ & $\left(L_{\odot}\right)$ & $4.8 \pm 0.3$ & $4.9 \pm 0.7$ & & $11.6 \pm 0.8$ & $13.1 \pm 2.4$ \\
$L_{\mathrm{FIR}} / M$ & $\left(L_{\odot} M_{\odot}^{-1}\right)$ & $0.5 \pm 0.1$ & $0.5 \pm 0.1$ & & $1.5 \pm 0.2$ & $1.4 \pm 0.3$ \\
\hline
\end{tabular}

Notes. The table lists the mean values obtained by averaging the medians of each Monte carlo simulation, for all cores in the whole Vela-D region. The columns labelled "mm-MIR" list the average values obtained using the SIMBA, BLAST and MIPS70 data in estimating the best-fit SED of each individual source. The columns labelled "BLAST" list the values obtained from BLAST photometry only. Only cores with $M>4.2 M_{\odot}$ (see Table 3 ) have been selected.

\subsubsection{Monte Carlo Simulations}

In this section, we want to discuss the effects that the errors on the physical parameters of each source, as determined using the procedure described in Section 4.2, have on the overall statistical distribution. To do this, we have used Monte Carlo simulations in which the values of temperature, mass, luminosity and luminosity-to-mass ratio of each source are varied within the uncertainties listed in Table 1. After each simulation, the histograms with the distribution of the four physical parameters are evaluated and the median values are determined. Also, the values of each bin in the histograms are stored for further computation. At the end of 500 such simulations, the mean and standard deviation of the median values are determined and listed in Tables 4 and 5.

In order to analyze possible systematic effects and biases, during each simulation the random values of temperature, mass, luminosity and luminosity-to-mass ratio were first determined based on the best fit results and uncertainties obtained using SIMBA, BLAST, and MIPS70 photometry (Section 4 and Table 1). Then, we repeated the procedure, but using the values obtained from BLAST-only photometry. Furthermore,
Table 5

Average Values from Monte Carlo Simulations for Cores in the SIMBA+MIPS70 Area

\begin{tabular}{lcrrrrr}
\hline \hline \multirow{2}{*}{ Physical Parameter } & \multicolumn{2}{c}{ Starless } & & \multicolumn{2}{c}{ Protostellar } \\
\cline { 3 - 4 } \cline { 7 - 7 } & & mm-MIR & \multicolumn{1}{c}{ BLAST } & & mm-MIR & \multicolumn{1}{c}{ BLAST } \\
\hline$T$ & $(\mathrm{~K})$ & $14.1 \pm 0.9$ & $13.1 \pm 1.0$ & & $15.3 \pm 0.2$ & $15.1 \pm 0.6$ \\
$M$ & $\left(M_{\odot}\right)$ & $7.4 \pm 1.2$ & $7.8 \pm 1.6$ & & $9.0 \pm 0.8$ & $9.7 \pm 1.2$ \\
$L_{\mathrm{FIR}}$ & $\left(L_{\odot}\right)$ & $5.0 \pm 0.9$ & $4.1 \pm 1.2$ & & $9.9 \pm 0.7$ & $10.1 \pm 1.7$ \\
$L_{\mathrm{FIR}} / M$ & $\left(L_{\odot} M_{\odot}^{-1}\right)$ & $0.8 \pm 0.3$ & $0.5 \pm 0.2$ & & $1.3 \pm 0.1$ & $1.1 \pm 0.2$ \\
\hline
\end{tabular}

Notes. Same as Table 4 for cores in the region of Vela-D covered by both the SIMBA and MIPS70 maps. In addition, like Table 4 only cores with $M>4.2 M_{\odot}$ have been selected.

the Monte Carlo simulations have been performed for two different sets of data: first, we used the complete sample of 141 Vela-D sources (see Table 4). Then, we repeated the simulations only for those BLAST cores falling in both the SIMBA and MIPS70 maps. In both cases, we selected only those cores with $M>4.2 M_{\odot}$ (see Table 5; see also note in Table 3), thus the values in these tables should be compared with Columns 4 and 7 in Table 3. In particular, Table 5 allows to evaluate the effects of our inability to correctly identify all protostellar cores outside the MIPS24 area (which overlaps well with the MIPS70 area, see Figure 3).

The results listed in Tables 4 and 5 can be summarized as follows: (1) in general, the uncertainties associated with the physical parameters obtained from BLAST-only photometry are higher, which is a direct consequence of the more accurate determination of the physical parameters achieved through $\mathrm{mm}$ to MIR photometry (when available); (2) the mean values of the physical parameters obtained from BLAST-only and mm-MIR photometry are consistent, within the errors, for both starless and protostellar cores, and separately in the two selected samples; (3) some differences may be seen between corresponding mean values of the whole sample and those of the smaller SIMBA/MIPS70 sample, which reflect several effects such as: 
completeness, correct identification of starless and protostellar cores outside the MIPS24 coverage (Section 5.4), different evolutionary stage of the densest, central region in Vela-D (Section 5.3.2); (4) despite these effects, the main features of starless and protostellar cores discussed in Sections 5.3.1 and 5.3.2 are still observable in Tables 4 and 5.

\subsection{Potential Effects Biasing the Distribution of Physical Parameters}

In Section 5.2, we mentioned that as many as $10 \%$ of the MIPS24 counterparts to the BLAST cores could be the result of chance associations. To estimate their potential impact on the distribution of physical parameters between starless and protostellar cores we used the following procedure. First, we randomly select $10 \%$ of the protostellar cores and assume that they are false associations, and thus transfer them into the sample of starless cores. Then, we estimate the median of all physical parameters using these re-defined samples of starless and protostellar cores. We repeat the previous two steps 500 times and finally we estimate the mean of all the median values previously determined. We thus obtain for starless and protostellar cores, respectively: $\langle T\rangle=14.5 \pm 0.2 \mathrm{~K}$ and $\langle T\rangle=15.8 \pm 0.1 \mathrm{~K} ;\langle M\rangle=$ $4.7 \pm 0.2 M_{\odot}$ and $\langle M\rangle=4.8 \pm 0.3 M_{\odot} ;\left\langle L_{\mathrm{FIR}}\right\rangle=4.4 \pm 0.1 L_{\odot}$ and $\left\langle L_{\mathrm{FIR}}\right\rangle=7.6 \pm 0.4 L_{\odot} ;\left\langle L_{\mathrm{FIR}} / M\right\rangle=1.0 \pm 0.1 L_{\odot} M_{\odot}^{-1}$ and $\left\langle L_{\mathrm{FIR}} / M\right\rangle=1.7 \pm 0.1 L_{\odot} M_{\odot}^{-1}$. We note that these average values are essentially equivalent to those shown in Table 3 and thus do not change any of our previous conclusions.

We also previously mentioned in Section 5.1 that outside of the region covered by MIPS24 we used MSX to separate starless and protostellar cores. It might be argued, then, that the trend we find in parameter space between starless and protostellar cores may be caused partially by our inability to detect faint MIR sources associated with BLAST cores using MSX data. However, we think this is not the case for two reasons: (1) if some of the cores outside the MIPS24 coverage are incorrectly classified as starless because of the lack of a $M S X$ counterpart, then the overall starless sample will be contaminated by (likely) warmer proto-stellar cores, thus reducing the difference in the median temperature between starless and proto-stellar cores; (2) within the MIPS24 map, when we consider a more complete sample $\left(M>4.2 M_{\odot}\right.$; see Figure 9$)$ the trend in physical parameter space discussed above is confirmed: the median temperature (or $L / M$ ratio) of starless and proto-stellar cores are, respectively, 14.2 and $15.4 \mathrm{~K}$ (or 0.9 and $1.4 L_{\odot} M_{\odot}^{-1}$, respectively). In addition, we note that $71 \%$ of the BLAST cores associated with a $M S X$ counterpart are concentrated in the region covered by MIPS24 (measuring about $1.8 \mathrm{deg}^{2}$ ), while the remaining $29 \%$ is found outside this area (which measures $\simeq 2.2 \mathrm{deg}^{2}$ ). Thus, using $M S X$ data alone, we find a larger density of candidate protostellar cores toward the central region of Vela-D, suggesting that this region is in a later stage of evolution. It also indicates that the number of BLAST cores with a MIR counterpart should be expected to decrease outside the MIPS24 map, which in turn makes the lack of sensitive MIR observations outside the MIPS24 area less critical. In fact, if we use the MSX source surface density as a rough indicator of the density of protostellar sources (correcting for the area ratio, 1.8/2.2, one expects a $\sim 25 \%$ probability of finding a protostellar core outside the MIPS24 area), and the ratio of the total number of MIPS24 sources to the number of MIPS24 sources with a MSX counterpart in the MIPS24 area $(\simeq 5)$, we thus estimate that the number of protostellar cores not detected by MSX outside the MIPS24 area is $\sim 10$.

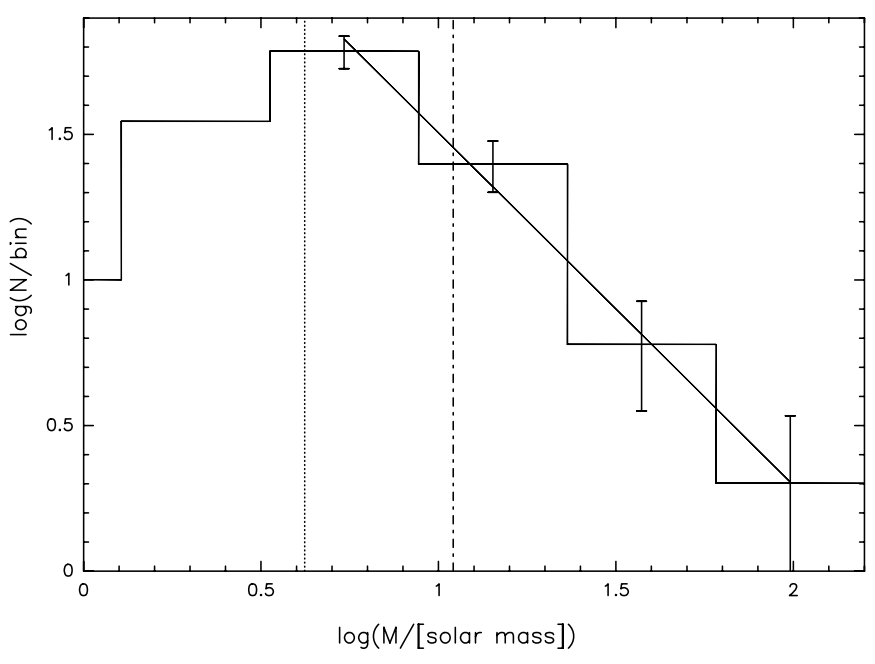

Figure 12. Combined starless and proto-stellar core mass function of the 141 BLAST objects associated with Vela-D. Poisson error bars are shown; however, additional sources of uncertainties are associated with the parameters of Equation (4). The masses in this plot are calculated assuming a dust emissivity index, $\beta=2.0$, a dust mass absorption coefficient, $\kappa_{0}=16 \mathrm{~cm}^{2} \mathrm{~g}^{-1}$ at $250 \mu \mathrm{m}$ a gas to dust ratio of 100 and a distance of $700 \mathrm{pc}$. The vertical dot-dashed line represents the mass completeness limit for sources warmer than $10 \mathrm{~K}$. However, because almost all of the Vela-D sources have temperatures $>12 \mathrm{~K}$ (Section 5.3) we also show the completeness limit for cores warmer than $12 \mathrm{~K}$ (dotted line). The solid line represents the best-fit to the CMF, yielding a power-law slope of $\alpha=-2.2$. The lowest bins suggest a turnover, but this shape is likely due to incompleteness. Likewise, the bin at $M \simeq 5.6 M_{\odot}$ is also likely to be somewhat affected by completeness.

\subsection{Mass Spectrum}

It is interesting to investigate the combined mass spectrum, including both starless and proto-stellar sources in Vela-D. The masses of individual cores were placed in logarithmically spaced bins and a lower limit on the error was estimated from the Poisson uncertainty for each bin. The resulting mass function is shown in Figure 12. We then fit a power law $\left(d N / d M \propto M^{\alpha}\right)$ to the $\mathrm{CMF}$ for $M>4 M_{\odot}$, finding a slope $\alpha=-2.2$ with a correlation coefficient $r=0.99$. The best-fit slope depends somewhat on the histogram binning. Given the relatively low number of sources in Vela-D, we could not vary greatly the bin widths; however, for bin widths of $\simeq 1.7$ to $4.2 M_{\odot}$ we obtain values $\alpha \simeq-2.1$ to $\alpha \simeq-2.5$, so we empirically assign the value $\alpha=-2.3 \pm 0.2$. We note that in the fit, the bin at $M \simeq 5.6 M_{\odot}$ has also been used, but because this bin is likely to be affected somewhat by completeness effects, it may be biasing low the resulting slope of the CMF.

Because almost all of the Vela-D sources have temperatures $>12 \mathrm{~K}$ (see Section 5.3) the appropriate mass completeness limit is shown as a vertical dotted line in Figure 12 for cores warmer than $12 \mathrm{~K}$. The two lowest bins suggest a turnover, but this shape is probably due to incompleteness and thus cannot be used to make any statement about the point at which the mass distribution flattens. Compared to previous surveys and measurements of the CMF, our data rely on a robust determination of the core temperature. There may be some concern over the use of a fixed value of the dust emissivity, $\beta$; however, as discussed by Chapin et al. (2008) this effect may shift the CMF, but does not affect the power-law index.

The slope of the CMF found for the cores in Vela-D is very similar to that determined for Orion $(\alpha=-2.35)$ by Nutter $\&$ Ward-Thompson (2006) in the mass range $M>2.4 M_{\odot}$, and also to that found for the cores in Perseus, Serpens, and 
Ophiucus $(\alpha=-2.3 \pm 0.4)$ by Enoch et al. (2008), in a comparatively lower mass range $\left(M>0.8 M_{\odot}\right)$. Our $\mathrm{CMF}$ is steeper than that found also in Vela-D by Massi et al. (2007), who report $\alpha \sim-1.9$ to -1.4 , and by Elia et al. (2007) who find $\alpha \sim-2.0$ to -1.3 (with $\alpha \sim-2$ as the most probable). The lower values for $\alpha$ measured by Massi et al. (2007) and Elia et al. (2007) are likely a consequence of the smaller source samples used to compute the CMF and also the fact that these surveys may have missed many of the coldest, low-mass sources. In fact, if we evaluate the CMF using only the BLAST cores with SIMBA counterparts we find values of $\alpha$ consistent with those of Massi et al. (2007) and Elia et al. (2007).

All of these CMFs appear to have remarkably similar shapes to that of the stellar IMF, as given for example by Kroupa (2002). While the observations taken together suggest that the CMF is a direct precursor of the stellar IMF, it should be noted that the measured masses of Nutter \& Ward-Thompson (2006), Enoch et al. (2008) and Massi et al. (2007) are somewhat more uncertain than ours because they lack (partially or completely) temperature information for the cores.

We have not attempted to evaluate separately the CMF of starless and protostellar cores in Vela-D, because of the relatively small number of sources in the two sub-samples. However, this has been done for the much larger source sample of the Vela deep map by Netterfield et al. (2009), who use this result to infer the lifetime of the BLAST starless cores in the VMR. Netterfield et al. (2009) find that cores above $14 M_{\odot}$ in the Vela deep map follow a CMF with $\alpha=-2.77 \pm 0.16$, which is moderately steeper than the CMFs mentioned earlier and also steeper than the Salpeter IMF. However, they also find that in the smaller Vela-C region, the CMF has $\alpha=-2.55 \pm 0.20$ thus consistent within the errors with the value found in Vela-D, also because, as previously mentioned, we may be biasing low the slope of the CMF. We note that the somewhat less steep CMF in Vela-D, which is in a later evolutionary phase compared to Vela-C, is consistent with cold cores following a steeper mass function than more evolved cores, as found by Netterfield et al. (2009).

\section{SUMMARY AND CONCLUSIONS}

In this paper, we have presented a detailed analysis of the dense cores in the Vela-D molecular cloud, utilizing the sensitive maps at 250, 350, and $500 \mu \mathrm{m}$ obtained by BLAST during its 2006 science flight from Antarctica. We have combined the multi-color BLAST photometry with previous MIR, FIR, and millimeter-continuum observations to determine the physical parameters of the population of cores detected by BLAST. Our sample includes a total of 141 objects, although only 89 cores are located within the common area covered by the MIPS 24 and MIPS70 maps. We summarize the other results of this paper as follows.

1. The multi-band photometry, combined with an isothermal modified blackbody model, allows us to analyze the effects that the uncertainties on the derived physical parameters of the individual sources have on the overall properties of starless and protostellar cores.

2. In the area of Vela-D covered by the MIPS24, MIPS70, and SIMBA maps we find 34 cores, out of 89 , having no MIPS24 or MIPS70 counterpart, with 28 of these cores not having been detected by SIMBA either. Therefore, these observations demonstrate the importance of observing the early cores at or near the SED peak, as made possible by BLAST.
3. The CMF determined from all (starless and protostellar) BLAST cores in Vela-D shows a power-law slope of $\alpha=-2.3 \pm 0.2$, consistent, within the errors, with other continuum (sub)millimeter surveys and in particular with the slope found by Netterfield et al. (2009) in Vela-C.

4. We find a moderate difference between the median mass of protostellar and starless cores. We also find that protostellar cores are somewhat warmer and more luminous than starless cores, and that there is a progressively higher fraction of protostellar cores with increasing core temperature. This suggests, as expected, a luminosity and temperature evolution due to the appearance of an embedded protostar.

5. In terms of the observational properties, there appear to be a smooth transition from the pre- to the protostellar phase, as suggested by differences found within the two populations. In particular, for protostellar cores we find a correlation between the MIPS24 flux, associated with the central protostar, and the temperature of the dust envelope. Our results can thus provide guidelines for understanding the physical conditions where the transition between preand protostellar cores takes place.

We acknowledge the support of NASA through grant numbers NAG5-12785, NAG5-13301, and NNGO-6GI11G, the NSF Office of Polar Programs, the Canadian Space Agency, the Natural Sciences and Engineering Research Council (NSERC) of Canada, and the UK Science and Technology Facilities Council (STFC). This work is also based, in part, on observations made with the Spitzer Space Telescope, which is operated by the Jet Propulsion Laboratory, California Institute of Technology under a contract with NASA. Support for this work was provided by NASA through an award issued by JPL/Caltech. L.O. acknowledges partial support by the Puerto Rico Space Grant Consortium and by the Decanato de Estudios Graduados e Investigación of the University of Puerto Rico.

\section{REFERENCES}

André, P., Ward-Thompson, D., \& Barsony, M. 2000, in Protostars and Planets IV, ed. V. Mannings, A. P. Boss, \& S. S. Russell (Tucson, AZ: Univ. Arizona Press), 59

Barnard, V. E., Vielva, P., Pierce-Price, D. P. I., Blain, A. W., Barreiro, R. B., Richer, J. S., \& Qualtrough, C. 2004, MNRAS, 352, 961

Beltrán, M. T., Brand, J., Cesaroni, R., Fontani, F., Pezzuto, S., Testi, L., \& Molinari, S. 2006, A\&A, 447, 221

Cao, Y., Terebey, S., Prince, T. A., \& Beichman, C. A. 1997, ApJS, 111, 387

Carey, S. J., et al. 2005, BAAS, 1252

Caswell, J. L., \& Haynes, R. F. 1987, A\&A, 171, 261

Chapin, E., et al. 2008, ApJ, 681, 428

De Luca, M., Giannini, T., Lorenzetti, D., Massi, F., Elia, D., \& Nisini, B. 2007, A\&A, 474, 863

Egan, M. P., Price, S. D., Shipman, R. F., Gugliotti, G. M., Tedesco, E. F., Moshir, M., \& Cohen, M. 1999, in ASP Conf. Ser. 177, Astrophysics with Infrared Surveys: A Prelude to SIRTF, ed. M. D. Bicay et al. (San Francisco, CA: ASP), 404

Egan, M. P., Shipman, R. F., Price, S. D., Carey, S. J., Clark, F. O., \& Cohen, M. 1998, ApJ, 494, L199

Elia, D., et al. 2007, ApJ, 655, 316

Enoch, M. L., Evans, II, N. J., Sargent, A. I., \& Glenn, J. 2009, ApJ, 692, 973

Enoch, M. L., Evans, II, N. J., Sargent, A. I., Glenn, J., Rosolowsky, E., \& Myers, P. 2008, ApJ, 684, 1240

Faustini, F., Molinari, S., Testi, L., \& Brand, J. 2009, A\&A, 503, 801

Fazio, G. G., et al. 2004, ApJS, 154, 10

Frisch, P. C., \& Slavin, J. D. 2003, ApJ, 594, 844

Giannini, T., et al. 2005, A\&A, 433, 941

Giannini, T., et al. 2007, ApJ, 671, 470

Griffin, M. J., et al. 2008, Proc. SPIE, 7010, 701006 
Helou, G., \& Walker, D. W. (ed.) 1988, Infrared Astronomical Satellite (IRAS) Catalogs and Atlases, Vol. 7: The Small Scale Structure Catalog (NASA RP-1190; Washington, DC: GPO)

Jijina, J., Myers, P. C., \& Adams, F. C. 1999, ApJS, 125, 161

Kirk, J. M., Ward-Thompson, D., \& André, P. 2005, MNRAS, 360, 1506

Kroupa, P. 2002, in ASP Conf. Proc. 285, Modes of Star Formation and the Origin of Field Populations, ed. E. K. Grebel \& W. Brandner (San Francisco, CA: ASP), 86

Lada, C. J., Muench, A. A., Rathborne, J., Alves, J. F., \& Lombardi, M. 2008, ApJ, 672, 410

Liseau, R., Lorenzetti, D., Nisini, B., Spinoglio, L., \& Moneti, A. 1992, A\&A, 265,577

Lorenzetti, D., Giannini, T., Vitali, F., Massi, F., \& Nisini, B. 2002, ApJ, 564, 839

Lorenzetti, D., Spinoglio, L., \& Liseau, R. 1993, A\&A, 275, 489

Massi, F., De Luca, M., Elia, D., Giannini, T., Lorenzetti, D., \& Nisini, B. 2007, A\&A, 466, 1013

Massi, F., Giannini, T., Lorenzetti, D., Liseau, R., Moneti, A., \& Andreani, P. 1999, A\&AS, 136, 471

Massi, F., Lorenzetti, D., \& Giannini, T. 2003, A\&A, 399, 147

Massi, F., Lorenzetti, D., Giannini, T., \& Vitali, F. 2000, A\&A, 353, 598

Massi, F., Testi, L., \& Vanzi, L. 2006, A\&A, 448, 1007

McKee, C. F., \& Ostriker, E. C. 2007, ARA\&A, 45, 565

Motte, F., André, P., \& Neri, R. 1998, A\&A, 336, 150

Motte, F., Bontemps, S., Schilke, P., Schneider, N., Menten, K. M., \& Broguiére, D. 2007, A\&A, 476, 1243

Murphy, D. C., \& May, J. 1991, A\&A, 247, 202

Netterfield, C. B., et al. 2009, ApJ, 707, 1824

Nutter, D., \& Ward-Thompson, D. 2006, MNRAS, 368, 1833
Pascale, E., et al. 2008, ApJ, 681, 400

Patanchon, G., et al. 2008, ApJ, 681, 708

Patanchon, G., et al. 2009, ApJ, 707, 1750

Rathborne, J. M., Lada, C. J., Muench, A. A., Alves, J. F., \& Lombardi, M. 2008, ApJS, 174, 396

Rieke, G. H., et al. 2004, ApJS, 154, 25

Simon, R., Rathborne, J. M., Shah, R. Y., Jackson, J. M., \& Chambers, E. T. 2006, ApJ, 653, 1325

Smith, R. J., Longmore, S., \& Bonnell, I. 2009, arXiv:0908.3910

Stahler, S. W., \& Palla, F. 2005, The Formation of Stars (New York: Wiley)

Strafella, F., et al. 2009, ApJ, submitted

Truch, M. D. P., et al. 2008, ApJ, 681, 415

Truch, M. D. P., et al. 2009, ApJ, 707, 1723

Turner, A. D., et al. 2001, Appl. Opt., 40, 4921

Vuong, M. H., Montmerle, T., Grosso, N., Feigelson, E. D., Verstraete, L., \& Ozawa, H. 2003, A\&A, 408, 581

Ward-Thompson, D., Motte, F., \& André, P. 1999, MNRAS, 305, 143

Whitney, B. A., Indebetouw, R., Bjorkman, J. E., \& Wood, K. 2004, ApJ, 617, 1177

Whitney, B. A., Wood, K., Bjorkman, J. E., \& Cohen, M. 2003, ApJ, 598, 1079

Wiebe, D. V. 2008, PhD thesis, Univ. Toronto

Williams, J. P., de Geus, E. J., \& Blitz, L. 1994, ApJ, 428, 693

Wu, J., Dunham, M. M., Evans, II, N. J., Bourke, T. L., \& Young, C. Y. 2007, AJ, 133,1560

Yamamura, I., Fukuda, Y., \& Makiuti, S. 2008, in AKARI/FIS-Release Note (Rev. 2; Noordwijk: ESA), http://www.sciops.esa.int/index.php? project $=$ ASTROF $\&$ page $=$ observers

Young, C. H., \& Evans, II, N. J. 2005, ApJ, 627, 293 